\begin{tabular}{|l|c|c|c|c|}
\hline $\begin{array}{l}\text { Cuadernos de Investigación Geográfica } \\
\text { Geographical Research Letters }\end{array}$ & 2020 & N $^{\circ} 46(2)$ & pp. 413-446 & eISSN 1697-9540 \\
\hline
\end{tabular}

\title{
DISTRIBUCIÓN ESPACIAL Y TEMPORAL DE GLACIARES, GLACIARES CUBIERTOS Y GLACIARES ROCOSOS DURANTE LA ÚLTIMA DEGLACIACIÓN EN EL VALLE DE LA BONAIGUA (PIRINEO CENTRAL)
}

\section{J. VENTURA-ROCA}

ANTALP Group (Antartic, Artic and Alpine environments), Universitat de Barcelona, España.

\begin{abstract}
RESUMEN. La aplicación del método paleogeográfico al estudio de geoformas glaciares y de glaciares rocosos permite su caracterización morfométrica y sedimentológica, el establecimiento de una detallada secuencia morfoestratigráfica y una propuesta cronológica para las fases glaciares identificadas. Este estudio analiza un total de 86 geoformas (57 depósitos glaciares, 21 glaciares rocosos y 8 lóbulos protalud) en el valle de la Bonaigua (cuenca de la Noguera Pallaresa, Pirineo Central) con especial atención a la diferenciación entre morrenas de glaciar cubierto y depósitos de glaciar rocoso. Otros temas estudiados referentes a glaciares rocosos son su origen periglaciar o glaciar; la posible funcionalidad actual de algunas geoformas y la detección de glaciares rocosos situados a altitudes bajas (en el actual entorno forestal) mediante la utilización de un modelo digital de elevación de alta resolución $(2 \times 2 \mathrm{~m})$ procedente de datos LIDAR. La hipótesis cronológica elaborada por correlación con otros altos valles pirenaicos (con dataciones absolutas disponibles) incluye 7 fases (6 fases glaciares y 1 fase periglaciar) en las que coexisten y/o evolucionan, en una dinámica paraglacial, glaciares, glaciares cubiertos y glaciares rocosos, y que situamos temporalmente entre finales del Dryas antiguo y la Pequeña Edad del Hielo (PEH).
\end{abstract}

Spatial and temporal distribution of glaciers, debris-covered glaciers and rock glaciers during the last deglaciation in the Valley of the Bonaigua (Central Pyrenees)

ABSTRACT. The application of the paleogeographicmethod to the studyof glacial landforms and rock glaciers allows their morphometric and sedimentological characterization, the establishment of a detailed morphostratigraphic sequence and a chronological proposal for the identified glacial phases. This study analyzes 86 landforms (57 glacial deposits, 21 rock glaciers and 8 protalus ramparts) in the Bonaigua Valley (Noguera Pallaresa Basin, Central Pyrenees), with special attention to the differentiation between debris-covered glaciers and rock glaciers. Other subjects studied concerning rock glaciers are: distinguish 
its glacial or periglacial origin; the possible current activity of some landforms, and the detection of rock glaciers located at low altitudes (in the current forest environment) through the use of high-resolution digital elevation model $(2 \times 2 \mathrm{~m})$ from LIDAR data. The chronological hypothesis elaborated by correlation with other high Pyrenean valleys (with absolute ages available) includes 7 phases (6 glacial phases and 1 periglacial phase) in which co-exist and/or evolve, in a paraglacial dynamic, glaciers, debris-covered glaciers and rock glaciers, and that we temporarily place between the end of the Oldest Dryas and the Little Ice Age.

Palabras clave: Pirineos, deglaciación, Dryas antiguo, Dryas reciente, fases glaciares holocenas, glaciares cubiertos, glaciares rocosos.

Key words: Pyrenees, deglaciation, Oldest Dryas, Younger Dryas, Holocene glacial stages, debris-covered glaciers, rock glaciers.

Recibido: 14 octubre 2019

Aceptado: 6 febrero 2020

Correspondencia: Josep Ventura Roca, ANTALP Group (Antartic, Artic and Alpine environments), Universitat de Barcelona, España.E-mail: jventuraroca2@gmail.com

\section{Introducción}

El estudio de las fases glaciares dentro de la última deglaciación (en este caso la pirenaica) ha sido objeto de creciente interés por parte de la comunidad científica en las últimas dos décadas. La incorporación en algunos estudios de dataciones por exposición cosmogénica (DEC), sobre superficies rocosas o bloques en los depósitos, permite la elaboración de una cronología general de la deglaciación. Este periodo se caracteriza por la alternancia de fases de intensa fusión glaciar junto con otras de claro avance glaciar (en especial en el Dryas antiguo, 17.5-14.5 ka y Dryas reciente, 12.9-11.7 ka), siempre localizadas estas en los altos valles y circos pirenaicos, donde la escasa presencia de depósitos que incluyan sedimentos lacustres y materia orgánica hace difícil el uso de otras técnicas de datación. Las fases de la deglaciación también se caracterizan por una gran variabilidad, coexistencia y superposición de depósitos glaciares y de lóbulos protalud o glaciares rocosos, caracterizados estos por primera vez en contexto pirenaico por Serrat (1979). Buena muestra del conocimiento sobre la deglaciación esta presentado en las recientes síntesis (para el conjunto de la Península Ibérica) de Palacios et al. $(2014,2016)$ sobre el Dryas antiguo, García-Ruíz et al. (2016) sobre el Dryas reciente, Oliva et al. (2016) sobre el patrón espacio-temporal de la periglaciación y Oliva et al. (2019) respecto a las fases glaciares durante en Cuaternario reciente. Por otra parte, el conocimiento de la deglaciación en la transición Pleistoceno-Holoceno y a lo largo de este periodo hasta la Pequeña Edad del Hielo (en adelante, $\mathrm{PEH}$ ) sigue siendo muy poco conocido a nivel general y especialmente a nivel pirenaico. Existen escasos estudios que identifican fases glaciales holocenas (Gellatly et al.,1992; García Ruiz et al., 2014; Fernandes et al., 2017) ocurridas ya 
al inicio del Holoceno o incluso en etapas más recientes (Holoceno medio, Holoceno tardío) con una extensión glaciar mayor que la desarrollada durante la fase histórica de la PEH.

El objetivo de este estudio es caracterizar las geoformas glaciares y de glaciares rocosos, el establecimiento de una secuencia morfoestratigráfica y la propuesta de una hipótesis cronológica para las fases glaciares de la deglaciación en el valle de la Bonaigua. La zona de este valle incluye, siguiendo el criterio de estudios anteriores (Ventura, 2017), cabeceras glaciares (con cumbres entre los 2700 y los $2900 \mathrm{~m}$ ) sin actividad glaciar durante la $\mathrm{PEH}$, pero que contienen suficiente volumen montañoso y altitud (fondos de circo por encima de los $2400 \mathrm{~m}$ ) para albergar una amplia variedad depósitos de otras fases glaciares holocenas anteriores y tardiglaciares.

\section{2. Área de estudio}

El valle de la Bonaigua (42 40'35'’ $\mathrm{N} / 42^{\circ} 35^{\prime} 32^{\prime \prime} / 0^{\circ} 58^{\prime} 42^{\prime \prime} \mathrm{E} / 1^{\circ} 05^{\prime} 04^{\prime \prime} \mathrm{E}$ ), valle afluente de la Noguera Pallaresa por su margen derecha, se localiza en la vertiente meridional del Pirineo Central (Fig. 1). Con una orientación general O-E se extiende a lo largo de $10 \mathrm{~km}$ entre el Port de la Bonaigua (2072 m), en el límite con el Val d'Aran, y el Pont de la Mola, en Sorpe (1258 m). Tiene una extensión de 40,06 km² de los cuales más del $60 \%$ se sitúan por encima de los $2000 \mathrm{~m}$ incluyendo numerosas cumbres con alturas superiores a los $2500 \mathrm{~m}$ (Pic de Bassiero, $2903 \mathrm{~m}$ ). En su cabecera destacan los altos circos compuestos de Gerber y Cabanes (ambos con $>7 \mathrm{~km}^{2}$ de superficie) y en su interior se conservan numerosas geoformas y depósitos glaciares. El valle de la Bonaigua se encuentra parcialmente dentro de la Zona Periférica de Protección del Parc Nacional d'Aigüestortes i Estany de Sant Maurici.

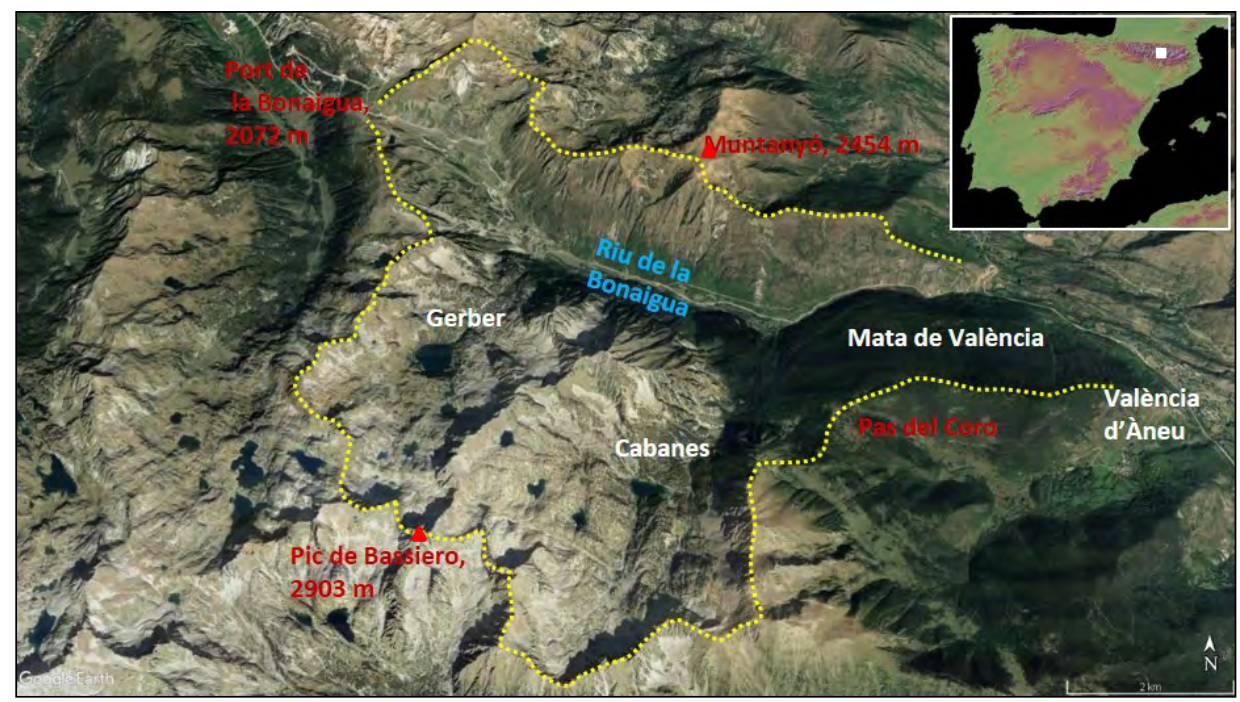

Figura 1. Localización de la zona de estudio en el Pirineo central (imagen 3D de Google Earth). 
El roquedo está formado por materiales pertenecientes a tres unidades de edad paleozoica (Institut Cartogràfic i Geològic de Catalunya-ICGC, 2007; Martín-Parra et al., 2016): a) rocas sedimentarias del Cambro-ordovícico y del Ordovícico superior (areniscas, pizarras y cuarcitas con intercalación de conglomerados cuarcíticos); b) rocas sedimentarias del Silúrico (pizarras negras) y del Devónico (calizas y pizarras). Estos materiales fueron afectados por el metamorfismo de contacto provocado por la intrusión de los granitoides de la Maladeta y Marimanha, con presencia, entre otras rocas, de mármoles y cuarcitas; y c) los granitoides de edad Carbonífero-Pérmico que se encuentran entre los $2000 \mathrm{~m}$ y las cumbres (circos de Gerber y Cabanes). Estructuralmente la zona forma parte de los "mantos inferiores" de edad alpina (ICGC, 2015); el manto del Orri al $\mathrm{S}$ y el de las Nogueres al N separados por la Falla Norte de la Maladeta, de edad Neógena (Ortuño, 2008).

El valle de la Bonaigua tiene, por su proximidad con el Valle de Arán, un régimen climático oceánico en el límite con el mediterráneo pirenaico occidental (Martin Vide et al., 2011). En el Port de la Bonaigua $(2260 \mathrm{~m})$ se registra una precipitación anual de $1232 \mathrm{~mm}$ y una temperatura media anual de $2,7^{\circ} \mathrm{C}$. La isoterma de $0^{\circ} \mathrm{C}$ se sitúa a los 2716 m (Fernandes et al., 2017).

\section{Antecedentes}

Las primeras referencias geomorfológicas sobre el glaciarismo en el valle de la Bonaigua las encontramos a mediados del siglo XIX, en la cita de Durocher (1844), que señala la existencia de rocas pulidas por el hielo entre Salardú y Esterri d Àneu, y las notas de Verneuil y Keyserling (1861) sobre morrenas situadas a 200-300 m por encima del fondo del valle. Nussbaum (1934), García Sainz (1935) y Solé Sabarís (1936) identifican depósitos glaciares (la Bonaigua de Baix, les Ares, la transfluencia glaciar del Pas del Coro) y delimitan la ocupación glaciar en la cuenca de la Noguera Pallaresa. Estos trabajos son revisados y ampliados posteriormente por Nussbaum (1956) y Zandvliet (1960). Fontboté et al. (1957), Hamelin (1958) y Masachs y Monturiol (1961), a raíz de la celebración en España del V Congreso del INQUA, presentan las primeras referencias a procesos y geoformas periglaciares, incluyendo también glaciares rocosos, y se cartografían varias morrenas y morrenas de nevero que posteriormente se han interpretado como glaciares rocosos (Gutiérrez y Peña, 1981). Los estudios más recientes del glaciarismo (Ventura, 1992; Ventura, 2010), las cartografías geomorfológicas publicadas a escala 1:50.000 y 1:25.000 de la zona por Serrat et al. (1994), Suárez Rodríguez y Alonso (2005), y Suárez Rodríguez (2016), y la cartografía geomorfológica realizada para sustentar este estudio, permiten una visión detallada de la geomorfología de la zona y de la extensión espacial del glaciarismo.

Los glaciares rocosos han sido abordados por varios autores; Gutiérrez y Peña (1981) en el circo de Gerber y el área de la Bonaigua, Ventura (1983) en el valle de Espot, Martí y Serrat (1990 y 1992) y Martí (1994) en el conjunto del Parque Nacional de Aiguiestortes. Las conclusiones comunes de estos autores son las siguientes: a) el dominio periglacial como principal generador de los glaciares rocosos con el soporte 
de la fracturación y los desprendimientos rocosos asociados; b) el periodo Tardiglaciar, frio y seco como época clave para su inicio generalizado, aunque esta pueda extenderse a fases anteriores y posteriores; c) la amplia franja altitudinal que ocupan (1700$2800 \mathrm{~m}$ ), la superposición de al menos 2 fases en un mismo glaciar rocoso y la variada colonización vegetal indican su formación en distintas pulsaciones frías; d) la posible coexistencia en el Tardiglaciar de glaciares rocosos junto a pequeños glaciares de circo; y e) la probable actividad actual de los situados en las cotas más elevadas. Ventura (2016) indica la potencial actividad de algunos glaciares rocosos y lóbulos protalud en la zona, con sus frentes a $>2.500 \mathrm{~m}$ al pie de paredes activas en los picos más elevados y en orientaciones N. Fernandes et al. (2017) en un inventario de glaciares rocosos y lóbulos protalud en los valles de Boí y Aran los incluye en tres generaciones, asociadas al proceso de deglaciación masiva entre el final del Último Máximo Glaciar (UMG) y el Holoceno.

\section{Metodología}

\subsection{Cartografía geomorfológica}

Del conjunto del valle de la Bonaigua se ha elaborado una cartografía geomorfológica, a escala 1:20.000 y a 1:10.000, de 5 zonas específicas, a partir de fotointerpretación de fotografías aéreas y ortofotos de alta resolución $(25 \mathrm{~cm})$ en color e infrarrojo color superponibles a mapas topográficos con las herramientas del servidor Vissir del ICGC, y completada con la consulta del visor 3D de Google Earth Pro. La dimensión mínima de las geoformas inventariadas ha sido $100 \mathrm{~m}$ (en longitud o anchura). El sistema cartográfico que utilizamos es el del Institut de Géographie et Durabilité (IGD) de la Universidad de Lausanne (Schoeneich, 1993; Pellitero, 2014; González Trueba, 2007). La cartografía realizada se encuentra en fase de delineación digital, una muestra gráfica de la misma aparece en la Figura 2. También se ha utilizado para la delimitación de glaciares rocosos fósiles en zonas forestales, un modelo digital de elevaciones (DEM) de resolución $2 \times 2 \mathrm{~m}$ generado a partir de datos LIDAR (ICGC, 2016), en presentación de sombreado mediante software QGIS. El trabajo de campo, se realizó entre los años 2010-2013 y 2018 (cartografía in situ, sedimentología de campo y validación final de la cartografía elaborada). 


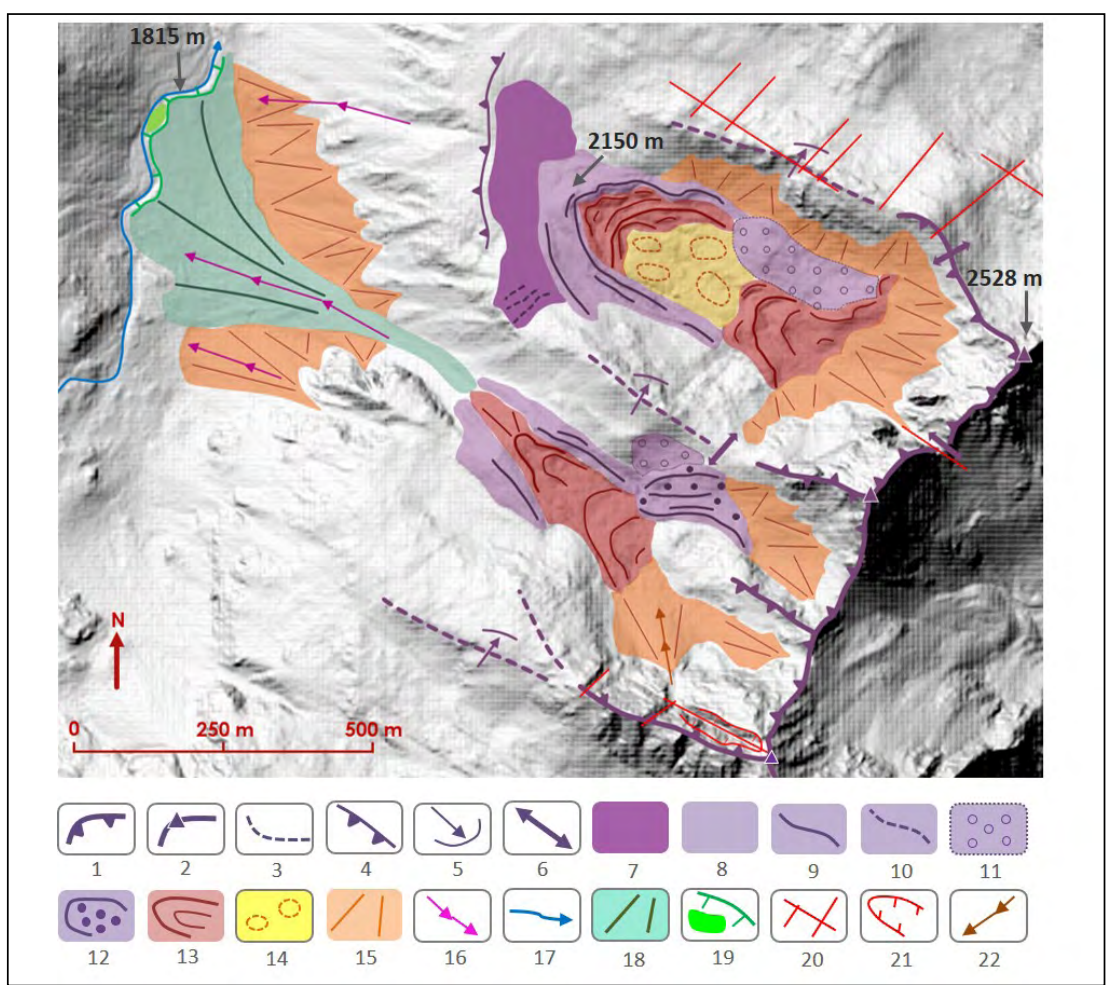

Figura 2. Cartografía geomorfológica de los circos de Es Paletes - Agulla de les Ares (Ruda).

Leyenda: 1. Circo glaciar; 2. Horn (pico); 3. Divisoria remodelada por la acción glaciar;

4. Hombrera; 5. Rocas aborregadas; 6. Difluencia glaciar; 7. Depósitos de till (inicio de la deglaciación); 8. Depósitos de till (fases de progresión dentro de la deglaciación); 9. Morrena; 10. Morrena degradada; 11. Till "open work"; 12. Morrena de glaciar cubierto; 13. Glaciar rocoso relicto; 14 . Acumulación de desprendimientos rocosos incorporados al glaciar rocoso; 15. Conos y taludes de derrubios; 16. Canal de aludes; 17. Curso fluvial; 18; Cono de deyección torrencial; 19: Relleno aluvial (T0) y escarpe fluvial; 20. Fracturación del macizo granítico; 21.

Cuenca de desprendimientos rocosos; 22. Canal de desprendimientos rocosos.

\subsection{Método paleogeográfico}

El método paleogeográfico (Scapozza, 2015) integra la morfoestratigrafía, que permite elaborar cronologías relativas sobre la base de las relaciones topológicas (sucesión espacial, continuidad, superposición, interrelación) entre geoformas y depósitos, así como el cálculo de parámetros paleoclimáticos (temperatura y precipitación). En las regiones de alta montaña, el método se basa en la morfoestratigrafía de los distintos estadios glaciares y de los glaciares rocosos (Scapozza, 2015). En los Pirineos se inicia este enfoque con los trabajos de Serrano et al. (2002) en el macizo de Posets, y Serrano y Agudo (2004) relacionando glaciares rocosos y deglaciación en los Pirineos aragoneses. 
La implementación de este método siguió las siguientes etapas:

- La cartografía geomorfológica detallada a nivel de cordones morrénicos y de glaciares rocosos.

- La numeración de todos los cordones morrénicos que permitan la reconstitución de una posición del frente glaciar y su combinación para definir las posiciones de referencia de cada paleoglaciar.

- La reconstrucción de la geometría de los paleoglaciares y el cálculo de la paleo altitud de la línea de equilibrio glaciar (del inglés, Equilibrium-Line Altitude, en adelante ELA) para cada fase diferenciada. Las ELA se han determinado mediante; a) el método "Toe-to-summit altitude method" (TSAM) que calcula la ELA como la media entre la cota del frente glaciar y la cota media de las cumbres del circo; y b) el método "Accumulation-area ratio" (AAR) con un AAR de 0,60 (Porter, 1975; Ben y Lehmkuhl, 2000; Serrano y González Trueba, 2004). En las delimitaciones de los paleoglaciares y la altimetría se han utilizado las bases cartográficas y las herramientas de edición del servidor Vissir del ICGC. La reconstrucción de la topografía de superficie se ha realizado manualmente siguiendo los criterios de concavidad en los circos y zonas de acumulación y convexidad en las lenguas y frentes glaciares (Wrethman, 2018). La variación o depresión de la ELA entre las fases glaciares (D-ELA) tiene como referencia la paleo ELA calculada para la PEH que en el cercano macizo de la Maladeta fue de $2870 \mathrm{~m}$ para los glaciares con orientación N (Lampre, 1994).

- La elaboración de una base de datos (Tabla 1) de los 21 glaciares rocosos y 8 lóbulos protalud inventariados siguiendo la clasificación de Monnier (2004).

- Para la datación relativa de los glaciares rocosos fósiles se ha utilizado el método desarrollado por Lambiel y Reynard (2003), que compara su posición con las zonas ocupadas por glaciares en distintos momentos del Tardiglaciar. Las correlaciones entre las distintas fases de glaciares rocosos se han basado en el cálculo del límite inferior del permafrost discontinuo (LIP) a partir de las altitudes inferiores de los frentes de los glaciares rocosos según orientaciones, y de la "depresión del permafrost" (DP) medida como la diferencia altitudinal media entre la base de los frentes de los glaciares rocosos en las diversas fases y el LIP actual (Haeberli, 1985; Barsch,1996; Scapozza y Mari, 2010). Se toma como referencia los actuales $2630 \mathrm{~m}$ y $2800 \mathrm{~m}$ como límites actuales del permafrost discontinuo para las vertientes N y S respectivamente (Serrano et al., 2009).

- El establecimiento con todos los depósitos inventariados de una "secuencia morfoestratigráfica compuesta", a partir de las secuencias locales establecidas en los circos de Gerber y Cabanes. 


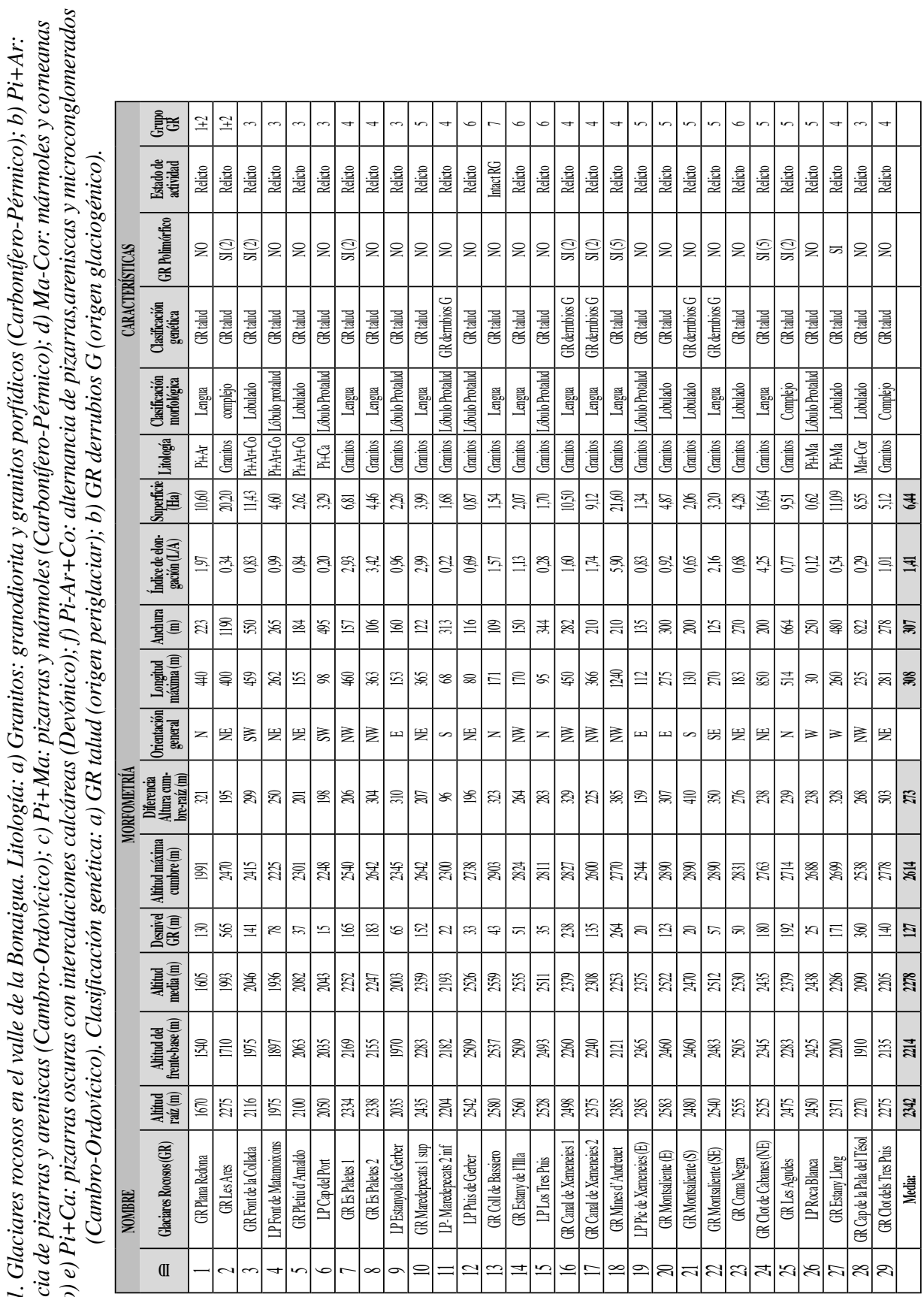




\section{Resultados}

\subsection{Análisis geomorfológico}

La elaboración del mapa geomorfológico a escala 1:20.000 ha permitido la identificación de 86 geoformas de acumulación: 57 depósitos glaciares organizados en 6 categorías (bloques erráticos, tills relacionados con las fases de extensión máxima, tills recientes relacionados con las fases de la deglaciación, tills "open work", morrenas y morrenas de glaciar cubierto), y 29 geoformas periglaciares entre glaciares rocosos, lóbulos protalud y morrenas de nevero. La numeración de los depósitos de los tipos genéticos comentados a continuación está referenciada en la Figura 3 y la Tabla 1.

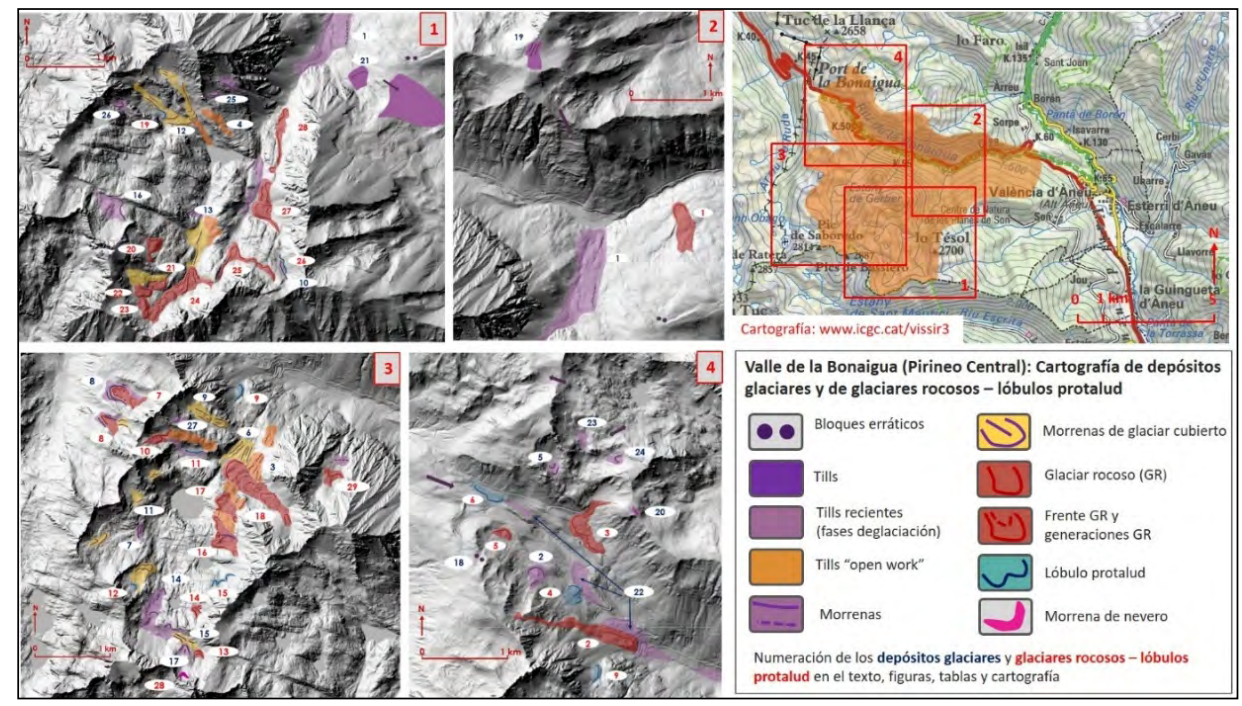

Figura 3. Valle de la Bonaigua: Cartografía de depósitos glaciares y de glaciares rocososlóbulos protalud.

\subsection{Depósitos glaciares}

Los depósitos relacionados con fases de máxima extensión glaciar han sido estudiados anteriormente (Ventura, 1992 y 2010). Solo se indican aquí aquellos nuevos depósitos localizados en el curso de este estudio y que sirven para completar la delimitación espacial de la fase de máximo glaciar y el inicio de la deglaciación: a) bloques erráticos en las cercanías del Port de la Bonaigua, a $2240 \mathrm{~m}$ en la zona de la Peülla (Fig. 3, punto18), indican una potencia mínima de $>170 \mathrm{~m}$ de la transfluencia glacial del Port de la Bonaigua; b) cordones morrénicos situados sobre la paleosuperfície de Cumials (Fig. 3, punto 20) y del Pic del Muntanyó (Fig. 3, punto 19) a $2454 \mathrm{~m}$ indican su funcionamiento como glaciar de plataforma; c) nuevas acumulaciones de bloques glaciares, placas de till y rellanos morrénicos se han identificado entre los 1300 y los $1660 \mathrm{~m}$ en la vertiente derecha del valle (bosque de la Mata de València). 
Los depósitos glaciares (Fig. 4) relacionados con la deglaciación son los siguientes:

a) Tills indiferenciados: Se trata de tills en general poco evolucionados, formados por bloques y cantos subangulosos y angulosos de granito y pizarras con una matriz limo-arenosa abundante de color ocre. Su situación preferente es en el fondo del valle y de circo. Los depósitos son los siguientes; a) el till más extenso se sitúa en el interior del valle de Cabanes (Fig. 3, punto 1) entre $1450 \mathrm{~m}$ (confluencia con el valle de la Bonaigua) y los $1800 \mathrm{~m}$ y en los laterales del valle, distinguiéndose algún cordón morrénico degradado, zonas con solo acumulaciones de bloques de grandes dimensiones y pequeños afloramientos de till basal con clastos estriados y abundante matriz lutítica de color gris azulado (Ventura, 2010); b) los situados en las cercanías del Port de la Bonaigua, (Fig. 3 , punto 22) entre 1700 y $2000 \mathrm{~m}$, presentan bloques graníticos en superficie en un entorno de roquedo no granítico; c) uno de estos depósitos, en las cercanías del Refugi de les Ares es fosilizado parcialmente por uno de los glaciares rocosos más antiguos de la zona (con frente a $1710 \mathrm{~m}$ ); d) al E del Tuc de la Cigalera, el fondo del circo $(2265 \mathrm{~m})$ modelado en terreno calcáreo está recubierto de till (Fig. 3, punto 23) con diversas depresiones en superficie (karts cubierto).

b) Morrenas de circo: Arcos morrénicos bien dibujados y morrenas frontolaterales se sitúan en la salida de los circos individuales o en interior de los grandes circos compuestos (Fig. 4 E): a) la franja altitudinal que ocupan se sitúa entre los 2000 y los $2600 \mathrm{~m}$; b) presentan dimensiones modestas con longitudes entorno a los 100-200 m (máximo de $350 \mathrm{~m}$ ) y alturas de 5-10 m; c) sobre los lomos morrénicos se distinguen bloques y cantos mayoritariamente angulosos y subangulosos embebidos en una matriz limo arenosa; d) pese a la escasa distancia a la que se encuentran de las paredes de los circos el volumen de los depósitos es notable, sobre todo en aquellos situados en orientaciones meridionales como por ejemplo en Clot de l'Estany Pudó (Fig. 3, punto 24), Coma de l'Àlber (Fig. 3, punto 5) y Pleta del Fils (Fig. 3, punto 25); e) en algunos circos se presentan en grupos formados por entre 2 y 4 arcos morrénicos próximos entre si cerrando en el interior a glaciares rocosos posteriores, como en los circos de Es Paletes (Fig. 3, punto 8) sobre el valle de Ruda o los situados en el Cap d'Estany Gerber (Fig. 3, puntos 14,15 y 17); f) se han cartografiado como morrenas de circo algunas geoformas que debido al mal estado de conservación, las reducidas dimensiones (longitud entorno a los $100 \mathrm{~m}$ ) y el contexto geomorfológico (150 m entre la cresta morrénica y la pared del circo) en el que se encuentran, también pudieran catalogarse como morrenas de nevero (protalus rampart o pronival rampart) siendo formas de transición entre morrenas y protalus rampart construidos por pequeños glaciares (glacierettes) adosados a las paredes de los circos (Fernandes et al., 2017; Whalley y Azizi, 2003; Whalley, 2009; Matthews et al., 2017) como se observa actualmente en los Pirineos en algunos pequeños glaciares funcionales durante la $\mathrm{PEH}$. Se encuentran ejemplos al SE de l'Agulla de les Ares (Fig. 3, punto 27) o al SE del Pic de Xemeneies (Fig. 3, punto 26), a 2340 y 2580 m respectivamente. 

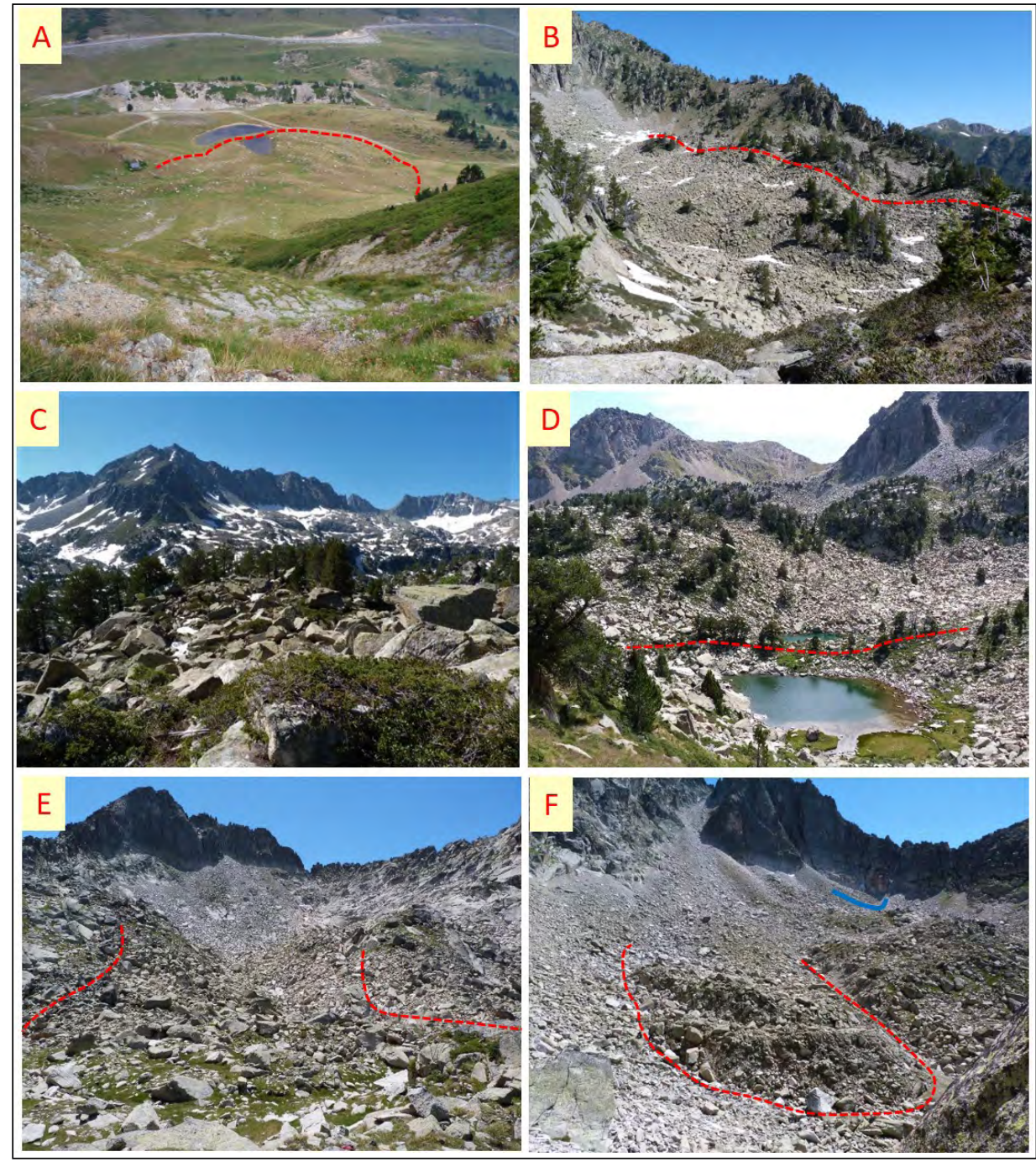

Figura 4. Ejemplos de depósitos glaciares y de glaciares rocosos. A: Glaciar rocoso relicto del Pletiu d'Arnaldo en la Bonaigua. Frente a 2063 m (Grupo GR-3, Fase 2); B: Zona interior del glaciar cubierto relicto de la Coma de Don Jaume en Gerber (Fase 3); C: Detalle de los tills "open work" situados (cota $2260 \mathrm{~m}$ ) en las divisorias internas del circo de Gerber (final Fase 2); D) Estanys de les Abadies (2280 m) en el Clot de Cabanes, rodeados de tills y morrenas de glaciar cubierto. Un cordón morrénico longitudinal (altura:8-10 m) separa a ambos lagos (final Fase 4); E: Morrenas fronto-laterales del glaciar de Montsaliente, cota 2590 m (Fase 6); F: Intact Rock Glacier del Coll de Bassiero (frente a 2537 m) y morrena de nevero de Bassiero (Grupo GR-7, Fase 7). 
c) Morrenas de glaciar cubierto: Se han clasificado como morrenas de glaciar cubierto (Fig. 4 B y D) a diversas geoformas catalogadas anteriormente como glaciares rocosos (Gutiérrez y Peña, 1981; Serrat et al., 1994; Suárez Rodríguez, 2016), sobre todo aquellas situadas en orientaciones meridionales. Los elementos diferenciadores utilizados en la revisión para la clasificación como glaciares cubiertos son; a) presencia de depresiones en cabecera (antiguos núcleos de hielo glaciar); b) deposito caótico, masivo y sin matriz (estructura "open work") formado por bloques métricos y cantos mayoritariamente angulosos (origen supraglacial), algunos de los cuales presentan un gran tamaño (diámetro de 3-8 m); c) morfología deprimida en su conjunto desde sus límites externos (morrenas) hacia el interior; d) se identifican morfologías como frentes bien definidos, lomas y surcos longitudinales paralelos al flujo glaciar (entre 130 y 350 m de recorrido y alturas de entre 4 y $15 \mathrm{~m}$ ) y depresiones de termokarst en el interior del depósito (Fernández-Fernández et al., 2017), algunas ocupadas por lagos. Ejemplos de estos lagos son el Estany Gerber de Baix (2121 m), los de Abadies (2279 m) y el del Pic de Serós (2384 m), formando este una depresión circular de $130 \mathrm{~m}$ de diámetro y hundido 3-12 m en la masa del depósito. En zonas más abiertas, alejadas de las paredes de los circos, se desarrollan facies de till de aspecto caótico con grandes bloques graníticos y una disposición en lomas longitudinales en la dirección del flujo glaciar, como en el Estany Gerber de Baix (Fig. 3, punto 6).

Los depósitos de glaciares cubiertos se sitúan en el valle de la Bonaigua entre los 2070 y los $2540 \mathrm{~m}$. Con superficies también variables (entre 9,5 y 74,4 ha), sus depósitos se encuentran dentro de una secuencia, que se repite a menudo, entre los tills, morrenas y los glaciares rocosos que los fosilizan. Al tratarse de geoformas fósiles no siempre es fácil su asignación genética y mucho menos cuando la geoforma en cuestión también presenta transiciones en distintas partes del depósito (de glaciar cubierto a glaciar rocoso). Ejemplos claros de esta tipología de glaciares cubiertos los encontramos en la Coma de Don Jaume (Fig. 3, punto 9), Pic de Serós (Fig. 3, punto 11) y Estanyola de Gerber (Fig. 3, punto 6) y de forma menos evidente con geoformas de transición en Pales de Nollerme (Fig. 3, punto12).

d) Tills "open work": Denominamos tills "open work" y los diferenciamos de las morrenas de glaciar cubierto, a aquellas extensas y densas acumulaciones de grandes bloques de aspecto caótico que se sitúan, dentro de los circos compuestos, sobre umbrales rocosos o en otras posiciones elevadas (Fig. 4 C y 5). En el VB encontramos estos depósitos exclusivamente en roquedo granítico, entre los 2100 y los $2400 \mathrm{~m}$, pero también se han observado en pizarras en la cuenca alta de la Noguera Pallaresa (Ventura, 2017). El contexto geomorfológico en que se ubican impide una asignación a caos de bloques de origen periglaciar. Los mejores ejemplos se encuentran a ambos lados de la entrada al circo de Gerber (Fig. 3, punto 3) y sobre el umbral rocoso que cierra el Estany Negre de Cabanes (Fig. 3, punto 4). 


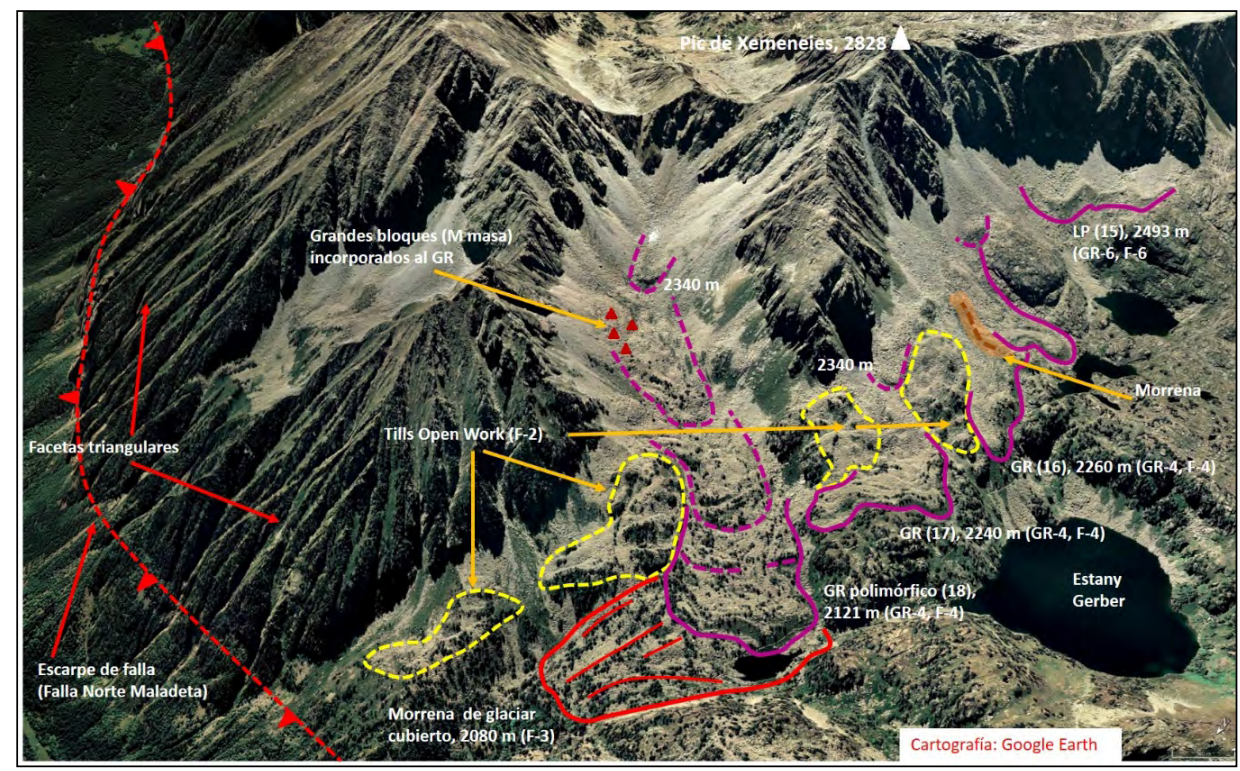

Figura 5. Imagen 3D del sector Mines d'Andreuet-Estany Gerber (Circo de Gerber). Depósitos glaciares (tills "open work", morrenas de glaciar cubierto, morrenas), glaciares rocosos (polimórficos) y lóbulos protalud. Se indica la cota $(m)$ de los frentes de las distintas geoformas y su asignación a las fases glaciares y de glaciares rocosos en el valle de la Bonaigua (cronología relativa).

En superficie, los tills “open work" muestran una morfología abultada, dominando los bloques de granito de dimensiones métricas, angulosos en su mayoría, con la presencia de escasos ejemplares subangulosos presentando caras pulidas y una aún más escasa matriz de arenas y gravas. De origen glaciar, depositados en posición supraglaciar y con escaso recorrido desde su área fuente, los relacionamos con episodios dentro de la deglaciación en los que los glaciares aún son extensos ocupando la totalidad de los grandes circos, en momentos de fuerte fusión glaciar y con una dinámica periglaciar intensa efectuada sobre crestas y sectores de las paredes liberadas por el hielo.

La diferenciación con las morrenas de glaciar cubierto se establece a partir de su ubicación y de las formas presentes en su superficie. En ocasiones se han confundido estos depósitos cartografiándose como GR. Lo cierto es que parte de estos depósitos son retrabajados posteriormente e incorporados a GR como por ejemplo al pie del Pic de Xemeneies $(2828 \mathrm{~m}$ ) donde orientadas al NW se suceden acumulaciones de till "open work" en las zonas elevadas y GR en las oquedades (Fig. 3, puntos 16 y 17).

\subsection{Glaciares rocosos y lóbulos protalud}

Los datos sobre glaciares rocosos están resumidos en la Tabla 1. Destacamos sus principales aspectos de localización, morfométricos y de clasificación: 
a) Altitud de los frentes: la altitud media de los frentes es de $2214 \mathrm{~m}$, situándose en una amplia franja altitudinal de casi $1000 \mathrm{~m}$ (1540-2537 m) indicadora de la existencia de varias fases de glaciares rocosos-lóbulos protalud. Los intervalos altitudinales mejor representados de los frentes son los de 2100-2300 m (10; $34 \%)$ y 2400-2600 m (9; 31\%) detectándose una marcada "fractura" entre el número de glaciares rocosos-lóbulos protalud situados por encima y por debajo de la cota $2100 \mathrm{~m}$ (21 y 8 respectivamente).

b) Orientación: los GR-LP situados al pie de paredes y vertientes con orientaciones de componente $\mathrm{N}(\mathrm{N}, \mathrm{NE}$ y NW) son las dominantes con 19 ejemplares (66\%), ocupando una superficie de 144,7 ha (78 \% de la extensión total). Se identifican en menor cantidad y superficie GR-LP en el resto de orientaciones (Fig. 6).

c) Litología: El $72 \%$ de los glaciares rocosos (21 de los 29) se localizan en terrenos graníticos y es un elemento relevante a pesar de la notable extensión en el valle de la Bonaigua de este roquedo, ubicado en las zonas más elevadas (>2000 m) con presencia de morfologías glaciares (circos, crestas, paredes, etc.) favorables a su desarrollo. Los escasos glaciares rocosos construidos en otros roquedos (básicamente pizarras y calizas), son de dimensiones menores (Fig. 4 A), presentan una morfología menos evidente (arcos, surcos y frentes menos marcados), están fosilizados en parte o remodelados por la dinámica periglaciar posterior y muestran una colonización vegetal casi completa (herbácea e incluso forestal). En la elevada densidad de glaciares rocosos que presenta el valle de la Bonaigua $\left(0,72\right.$ por $\left.\mathrm{km}^{2}\right)$, la litología granítica tiene una parte importante en su explicación. Este control litológico de los granitos en el Pirineo es similar al ejercido por las cuarcitas en la distribución y densidad de glaciares rocosos en la Cordillera Cantábrica (Gómez-Villar et al., 2011).

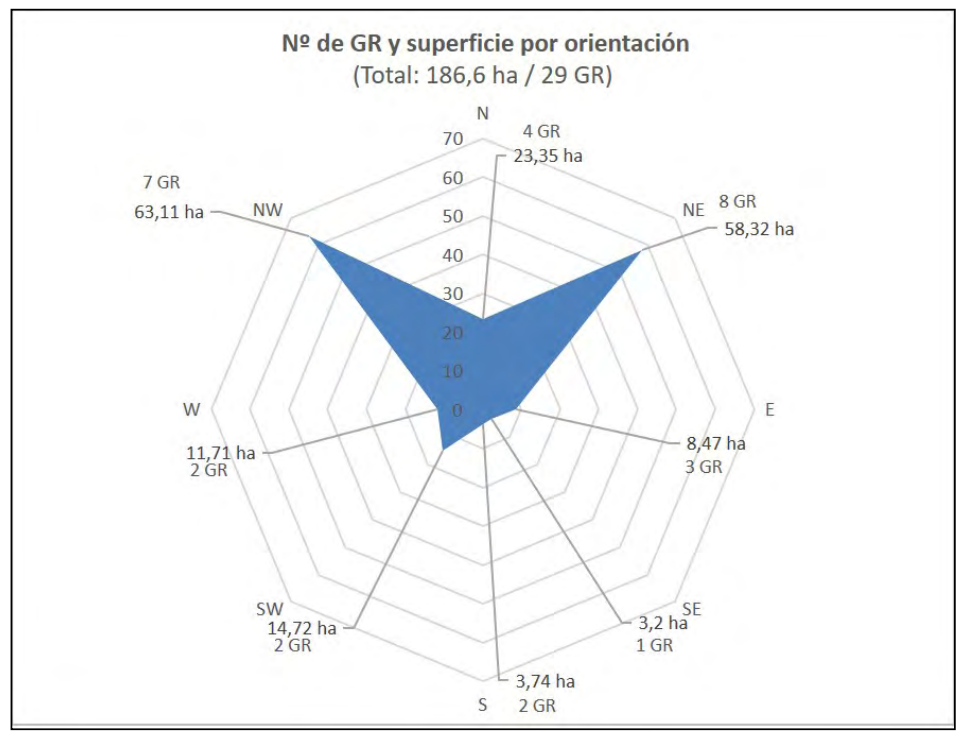

Figura 6. Glaciares rocosos y superficie por orientación. 
d) Localización / Área fuente: Son mayoritarios los glaciares rocosos-lóbulos protalud que se sitúan en la prolongación de taludes de derrubios (24 GR; 83 \%) y en menor medida los glaciares rocosos generados total o parcialmente desde depósitos morrénicos, ya sean morrenas o tills "open work" (5; $17 \%)$. Ejemplos de este segundo grupo son los situados al SE del Pic de Montsaliente en Cabanes (Fig. 3 y Tabla 1, puntos 21, 22).

e) Longitud (L) - anchura (A): La longitud media es de $308 \mathrm{~m}$ (468 m para los glaciares rocosos en lengua) con unas longitudes comprendidas entre $30 \mathrm{~m}$ (Fig. 3 y Tabla 1, punto 26) y $1240 \mathrm{~m}$ (Fig. 3 y Tabla 1, punto 18). Solo tres de ellos tienen una longitud superior a los $500 \mathrm{~m}(10 \%)$, la gran mayoría está en dimensiones de $<500$ m (26; $90 \%)$, siendo básicamente los lóbulos protalud los situados por debajo de los $<100 \mathrm{~m}$ de longitud. La anchura media es de $307 \mathrm{~m}$ (400 m para los glaciares rocosos lobulados) con unas dimensiones comprendidas entre los $116 \mathrm{~m}$ y $1190 \mathrm{~m} ; 20$ ejemplares de $<300 \mathrm{~m}$ (69\%) y 9 ejemplares de $>300 \mathrm{~m}$ (31\%), donde solo 4 de ellos presentan una anchura superior a los $500 \mathrm{~m}$. Los datos sobre el índice de elongación (L/A) indican una distribución de glaciares rocososlóbulos protalud donde dominan los glaciares rocosos lobulados $(17 ; 59 \%)$ incluyendo en este grupo a todos los LP, sobre los GR en lengua $(12 ; 41 \%)$ sin alejarse de las proporciones estándar de repartición entorno al $50 \%$. Glaciares rocosos en lengua, bien definidos a partir de una $L / A=1,5$, se encuentran 10 ejemplares, 3 de los cuales presentan un índice L/A $>3$ (Fig. 3 y Tabla 1, puntos 18,24 y 8 ).

f) Superficie: La extensión total ocupada por glaciares rocosos y lóbulos protalud es de 186,6 ha. Su extensión media es de 6,4 ha, aunque esta cifra queda distorsionada por la superficie de los escasos pero grandes glaciares rocosos. El grupo mayor lo componen los 17 aparatos de reducidas dimensiones ( $<5$ ha; $59 \%$ ) ocupando solo el $24 \%$ de la superficie total (45 ha). Un grupo intermedio de 5 aparatos se sitúa entre 5 y 10 ha (17\%) ocupa a 39 ha de la superficie total (21\%), mientras que los 7 grandes glaciares rocosos con más de 10 ha (24\%) ocupan el $55 \%$ de la superficie (102 ha). El más extenso es el Mines d'Andreuet (Figs. 3 y 5, Tabla 1, punto 18) con 21,6 ha de superficie. Casi todos los glaciares rocosos de este último grupo son del tipo polimórfico, es decir, construido por sucesivas generaciones de ellos. En la Figura 7 se muestra la relación entre franjas altitudinales (altitud de los frentes de glaciares rocosos) y superficie ocupada. 


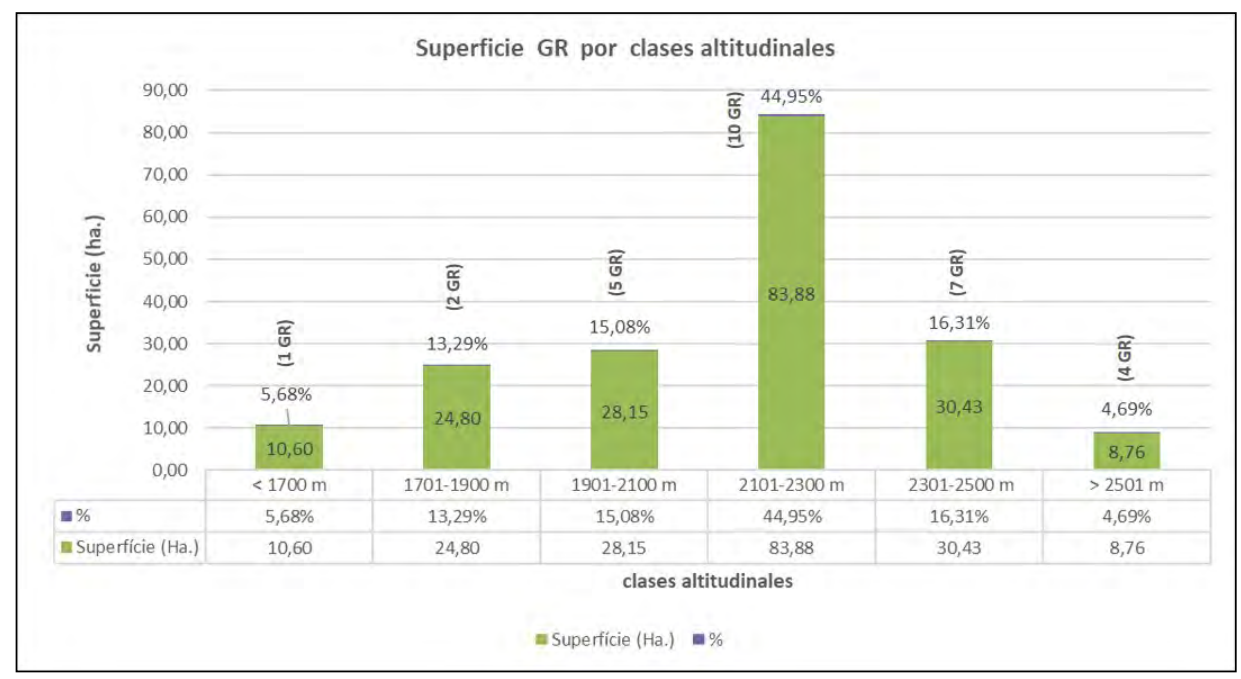

Figura 7. Glaciares rocosos, superficie por clases altitudinales.

g) Clasificación morfológica: Se identifican 11 glaciares rocosos en lengua (38\%), 7 lobulados (24\%), 3 complejos (10\%) y 8 lóbulos protalud (28\%) (Fig. 8)

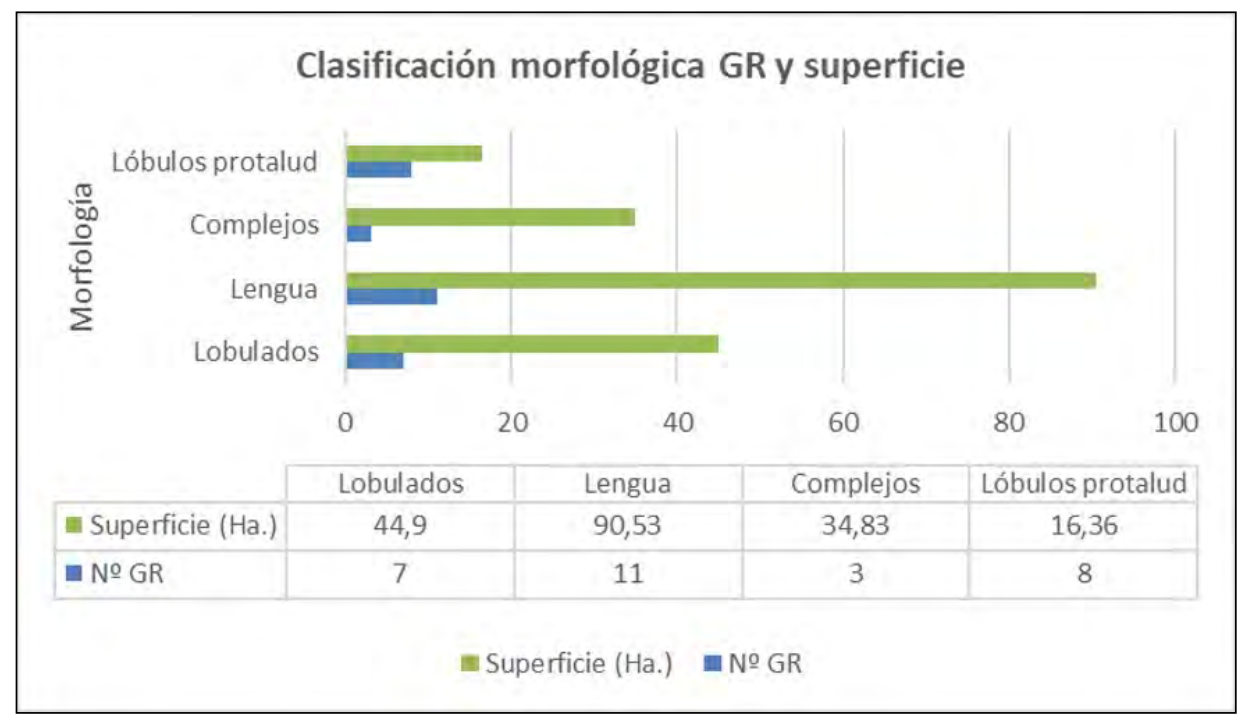

Figura 8. Clasificación morfológica de los glaciares rocosos y superficie.

h) Clasificación genética: La gran mayoría de los glaciares rocosos $(24 ; 82,7 \%)$ tienen su génesis a partir de talud de derrubios (origen periglaciar) frente a 5 
de ellos $(17,3 \%)$ generados a partir de depósitos morrénicos (glaciares rocosos glaciogénicos).

i) Glaciares rocosos monomórficos y polimórficos: 9 (31\%) de los 29 glaciares rocosos en el valle de la Bonaigua son del tipo polimórfico (Frauenfelder y Kääb, 2000) y se corresponden con los de mayor extensión. El número de "generaciones" internas oscila entre 2 y 5 , presentando variaciones en los frentes internos, tanto en distancia entre ellos (entre 100 y $400 \mathrm{~m}$ ) como en la diferencia en altura a la que se encuentran (entre $16 \mathrm{~m}$ y $235 \mathrm{~m}$ ). El cuerpo de derrubios de cada generación no solo se identifica por un lóbulo frontal bien dibujado, sino también por cambios en la morfología superficial de arcos y surcos, tamaño de los clastos y masividad del conjunto. Incluidos dentro del cuerpo de un glaciar rocoso son frecuentes las acumulaciones caóticas de bloques enormes (5-15 m de diámetro) procedentes de grandes desprendimientos en las paredes de los circos.

j) Clasificación por actividad: 28 de los 29 glaciares rocosos son relictos o fósiles y solo uno, el de Coll de Bassiero (Fig. 3 y 4 F y Tabla 1, punto 13) se clasifica como "intact rock glacier". Esta categoría, que incluye a los glaciares rocosos activos e inactivos (Barsh, 1996; Lilleoren y Etzelmüller, 2011; KellererPirklbauer et al., 2012), atiende su adscripción a criterios geomorfológicos (frente abrupto y con presencia de sedimentos de granulometría fina, formas netas en frente, arcos y surcos, enlace con talud activo, bloques con aristas vivas y mínima o nula cubierta vegetal) y de contexto morfotopográfico (orientación $\mathrm{N}$, cumbres a $>2800 \mathrm{~m}$ y frentes a $>2500 \mathrm{~m}$, dimensión y fracturación en las paredes del área fuente) y asume la permanencia de núcleo de permafrost en ellos.

\section{Discusión}

\subsection{Fases glaciares y glaciares rocosos}

A partir de la información recogida en la Figura 3 y la Tabla 1 y la aplicación del método paleogeográfico, se han identificado varias fases glaciares en el contexto de la deglaciación en el valle de la Bonaigua, que se sintetizan en la Tabla 2 y en la Figura 9. Para ello se han elaborado dos secuencias morfoestratigráficas locales, una en el circo de Gerber y otra en el circo de Cabanes, y una secuencia compuesta para ambas zonas que se presenta en la Figura 10. Esta secuencia morfoestratigráfica recoge la información sobre las morrenas en función de sus características morfológicas y sedimentarias, su altitud y la distancia al fondo del circo. También incluye la posición de los distintos glaciares rocosos y su relación con las morrenas y los datos elaborados de D-ELA (variación de la ELA entre cada fase y la ELA de referencia de la PEH) y de la DP (variación del límite inferior del permafrost entre los frentes de los glaciares rocosos de cada fase y el de referencia). 


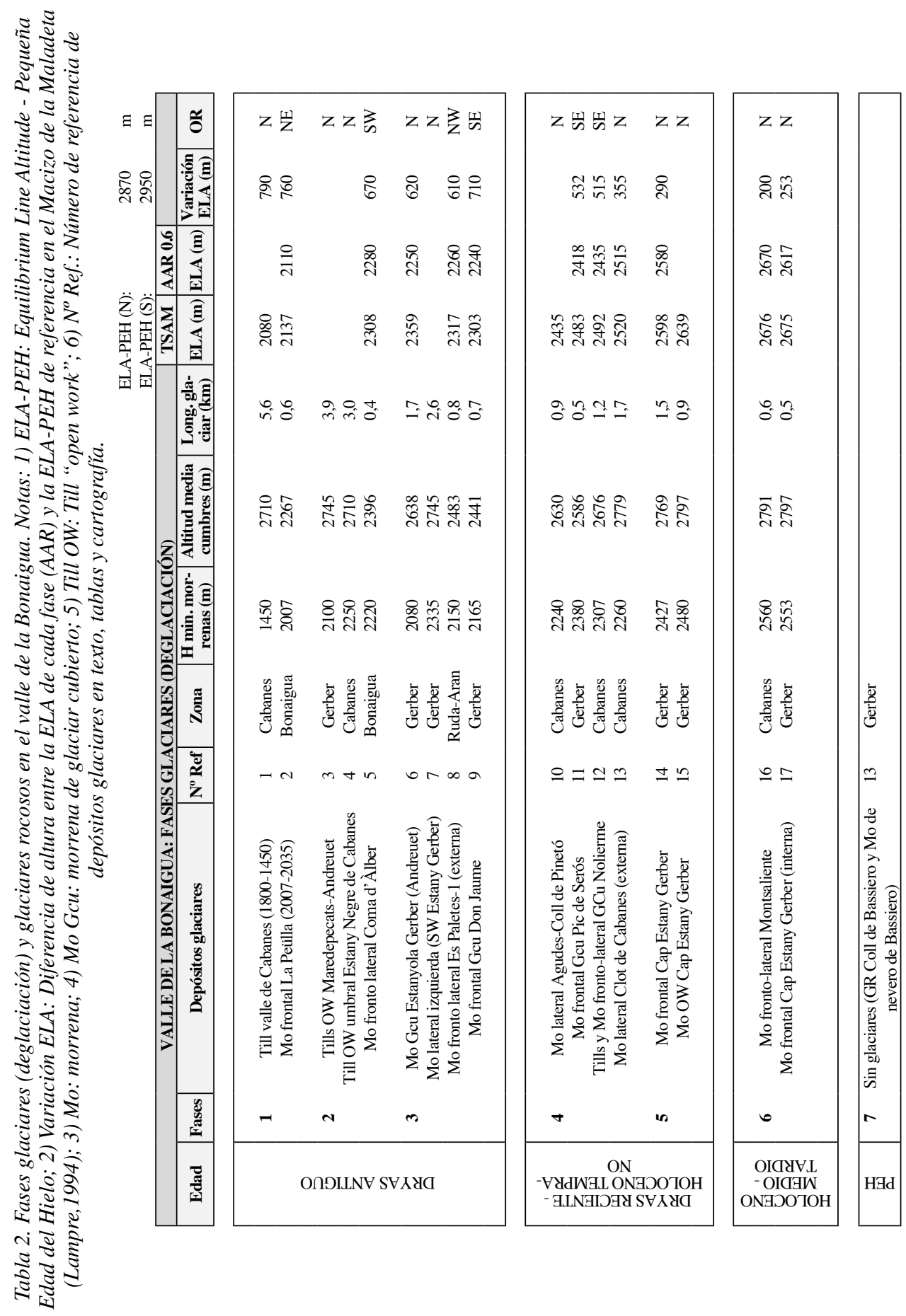




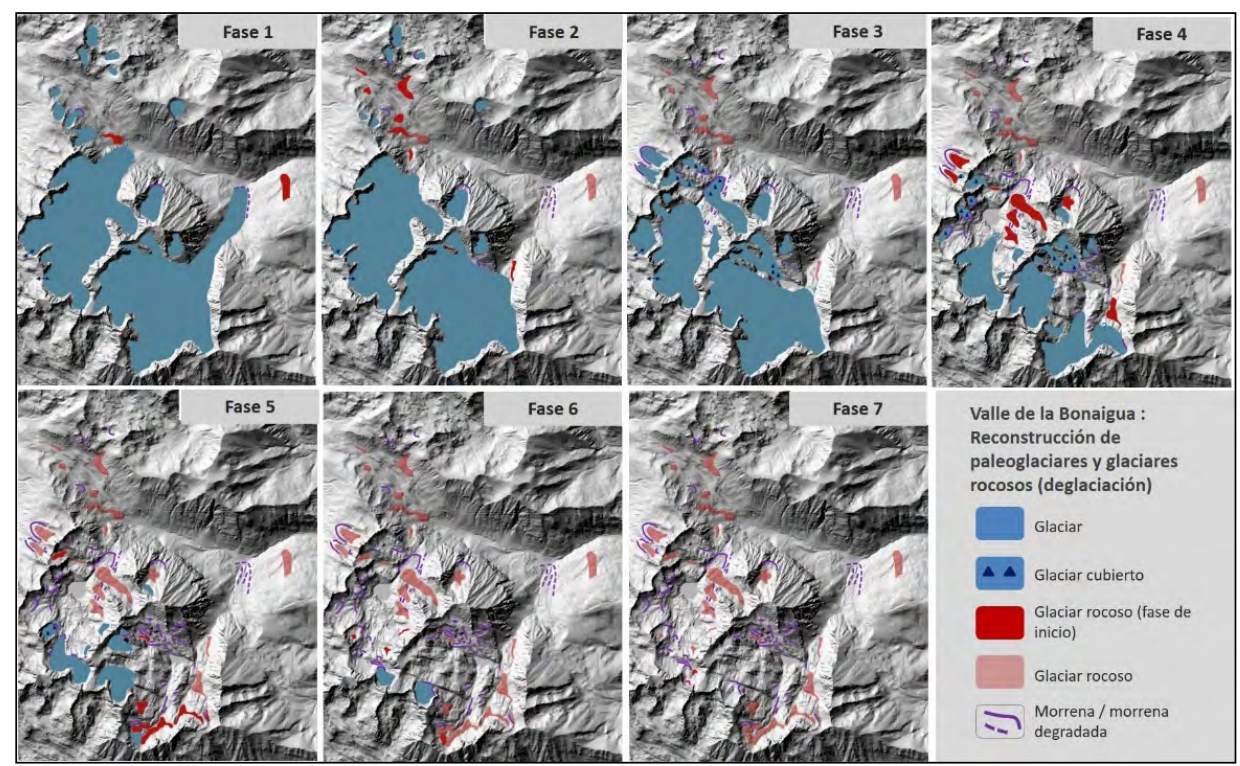

Figura 9. Reconstrucción de paleoglaciares y glaciares rocosos por fases (deglaciación) en el valle de la Bonaigua (Pirineo Central).

El punto de partida para situar las fases glaciares de la deglaciación en el valle de la Bonaigua es el mapa de la ocupación glaciar de la zona durante el Último Ciclo Glaciar (Fig. 11). El valle está ocupado por una lengua glaciar de $10 \mathrm{~km}$ de longitud que confluye con el glaciar principal de la Noguera Pallaresa (530 m de potencia en la zona de confluencia, que se convierten en $+800 \mathrm{~m}$ en la cubeta de Esterri d'Àneu). Alimentan al glaciar de la Bonaigua los hielos procedentes de los grandes circos de Gerber y Cabanes, de los circos colgados de Estany Pudó, Clot d'Arts y Clot dels Tres Puis, del glaciar de plataforma del Pic del Muntanyó y de la transfluencia glaciar del Port de la Bonaigua (con hielos procedentes del glaciar de la Garona). En el máximo glaciar el hielo supera la divisoria con el vecino valle de Son, generando una transfluencia glaciar con importantes depósitos morrénicos. Otros depósitos glaciares (obturación del Planell de Breviari, tills de la Mata de València, etc.) indican fases glaciares al final del UMG de menor potencia $(+300 \mathrm{~m})$ pero manteniendo la conexión del glaciar de la Bonaigua con los glaciares de la Noguera Pallaresa y de la Garona (Ventura, 1992; Ventura, 2010). Las fases son las siguientes:

Fase 1: a) En la primera fase identificada el valle de la Bonaigua está libre de hielo; b) glaciares extensos ocupan la totalidad de los grandes circos emitiendo lenguas hacia el valle de la Bonaigua (Cabanes, 5,6 km; Gerber, 4,6 km); c) pequeños glaciares de circo se mantienen en los macizos menos elevados $(<2500 \mathrm{~m})$ en la Peülla y Tuc de la Cigalera; d) paleo ELA a 2110 m (diferencia ELA de 790 m en relación a la $\mathrm{PEH}$ ); e) desarrollo de los primeros glaciares rocosos en la zona (grupos 1 y 2 ) en laderas del valle orientadas al $\mathrm{N}$ y a cotas bajas $(<1700 \mathrm{~m}$; 
DP de $1000 \mathrm{~m}$ ) en áreas ocupadas por el hielo al final del UMG. El frente del glaciar rocoso de les Ares (Fig. 3 y Tabla 1, punto 2) se superpone a tills depositados desde la transfluencia glaciar del Port de la Bonaigua, no funcional ya durante esta fase.

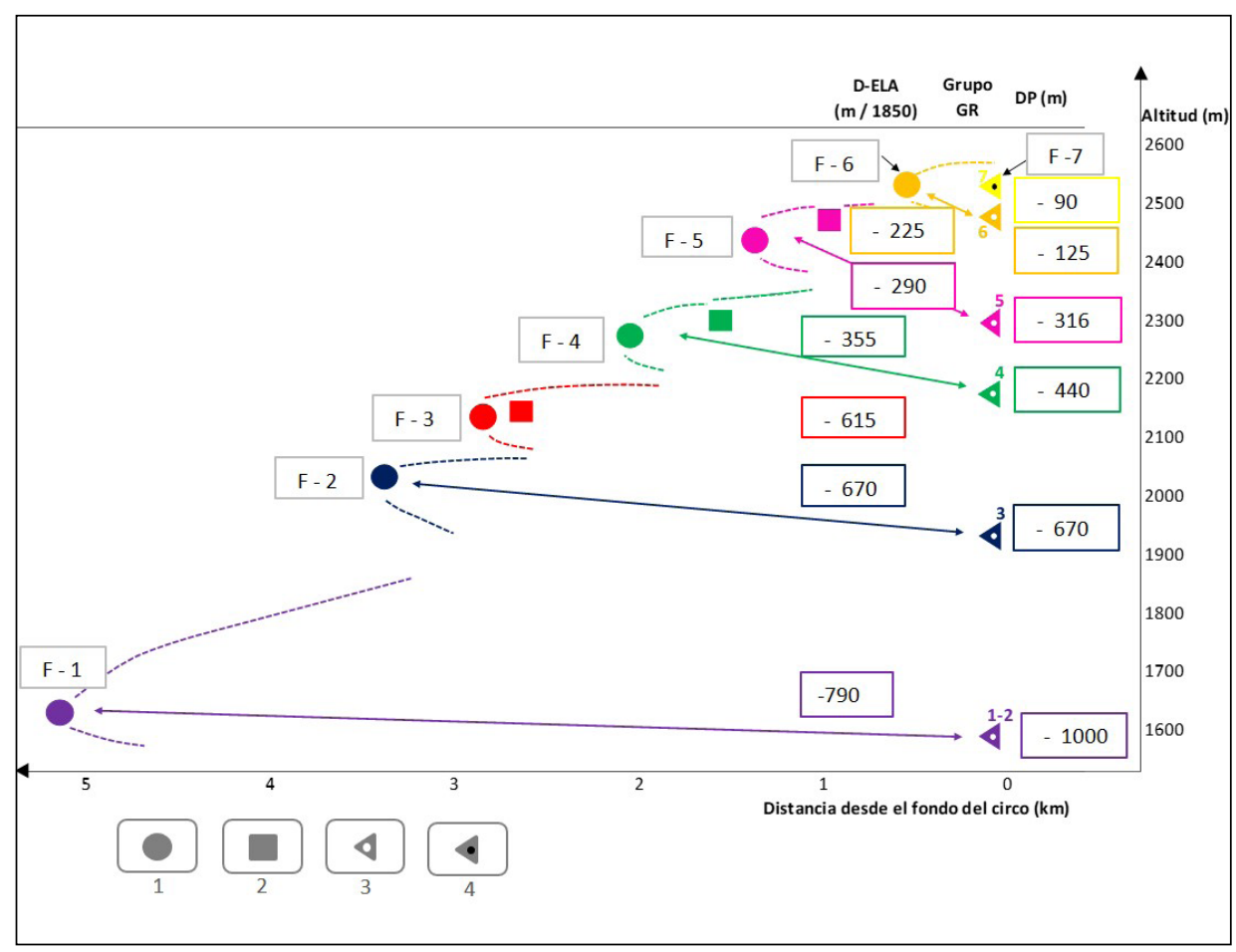

Figura 10. Secuencia morfoestratigráfica compuesta de la deglaciación en la vertiente $N$ del valle de la Bonaigua (circos de Gerber y Cabanes). Leyenda: 1- Morrena; 2- Morrena de glaciar cubierto; 3- Glaciar rocoso (relicto); 4- Glaciar rocoso (Intact rock glacier); D-ELA: Variación de la ELA en relación a la ELA de la PEH $(2870$ m, orientación $N)$ DP: Variación del permafrost en relación al LIP actual $(2630 \mathrm{~m}$, orientación $N)$.

Fase 2: a) después del retroceso de los glaciares de Gerber y Cabanes al final de la Fase 1, los glaciares de la Fase 2 se estabilizan situando sus frentes a 2050-2100 m, ocupando aún la totalidad de los grandes circos compuestos (ELA a $2200 \mathrm{~m}$ y diferencia ELA de $670 \mathrm{~m}$ ) pero sin emitir lenguas glaciares (desaparece la lengua glaciar del valle de Cabanes); b) los pequeños glaciares de circo en el entorno del Port de la Bonaigua son de reducidas dimensiones con frentes estabilizados en morrenas fronto laterales voluminosas a 2200$2300 \mathrm{~m}$, en las que se distingues varios cordones morrénicos muy próximos entre sí; c) aparecen nuevos glaciares rocosos (grupo 3) situados en las zonas recientemente liberadas por los hielos en la salida de los grandes circos y sobre todo en las vertientes ( $\mathrm{N}$ y $\mathrm{S}$ ) próximas al Port de la Bonaigua (con frentes entre 
1910 m-2063 m; DP de 670 m); d) al final de la Fase 2 se produce un notable retroceso glaciar que propiciará la sedimentación de importantes volúmenes de till "open work" situados en posiciones elevadas sobre umbrales y barras rocosas en el interior de los grandes circos y la fragmentación de estos en diferentes aparatos glaciares independientes.

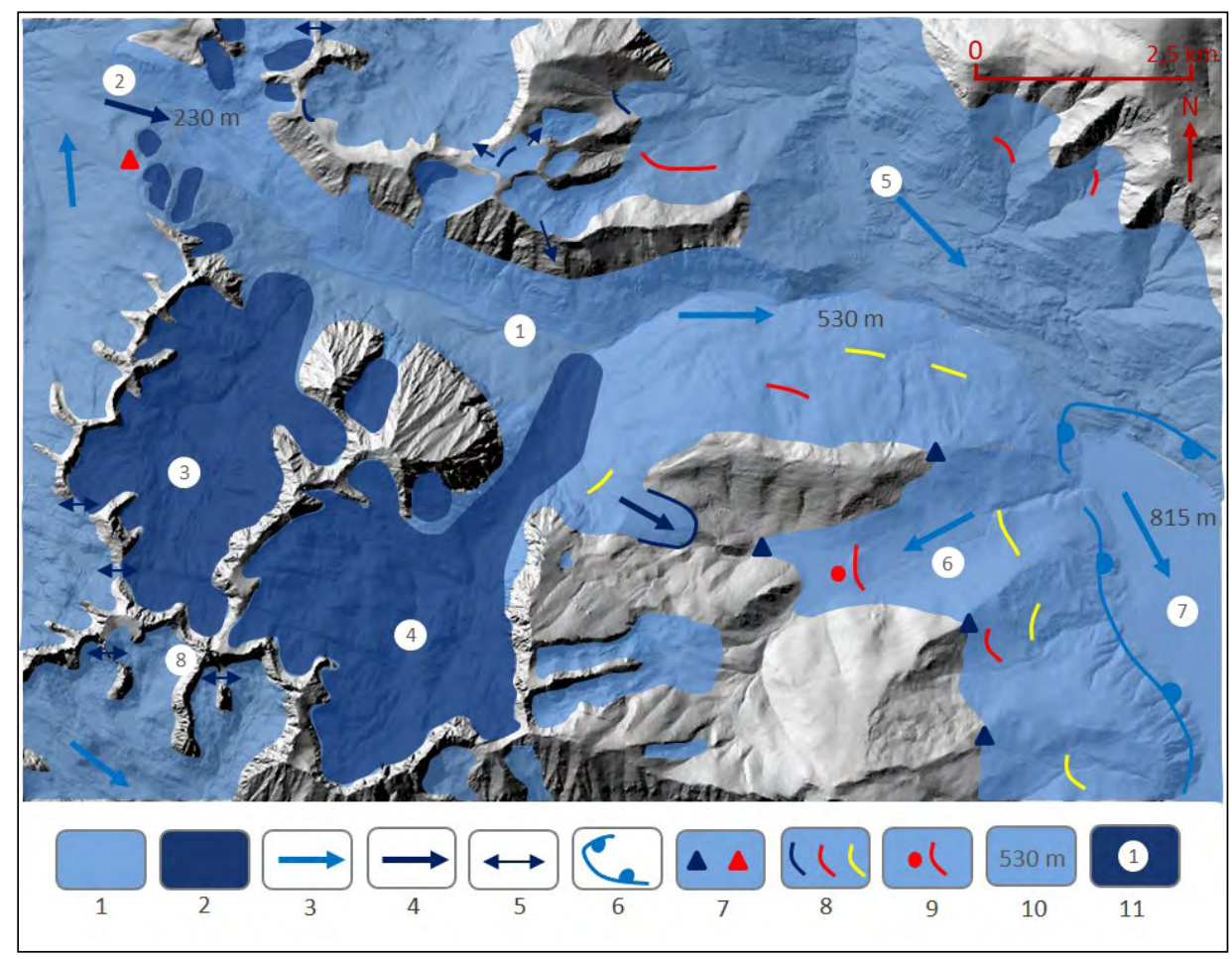

Figura 11. Reconstrucción de la ocupación glaciar durante el Último Ciclo Glaciar en el entorno del valle de la Bonaigua. Leyenda: 1. Extensión glaciar máxima en el Último Ciclo

Glaciar; 2. Extensión glaciar durante la F-1 de la deglaciación del valle de la Bonaigua; 3. Dirección del flujo glaciar; 4. Transfluencia glaciar; 5. Collado de difluencia glaciar; 6. Cubeta de sobreexcavación glaciar; 7. Bloques erráticos de las fases de máximo glaciar (azul) y de estabilización post-máximo (rojo); 8. Morrenas y depósitos yuxtaglaciares de las fases de máximo glaciar (azul), estabilización post-máximo (rojo) y del último máximo glaciar, UMG (amarillo); 9. Obturación glaciolacustre (fase de estabilización post-máximo); 10. Potencia del hielo en los glaciares; 11. Toponimia. Toponimia: 1. Paleoglaciar de la Bonaigua; 2. Port de la Bonaigua (2072 m); 3. Circo de Gerber; 4. Circo de Cabanes; 5. Paleoglaciar de la Noguera Pallaresa; 6. Valle de Son; 7. Cubeta de Esterri d'Àneu (950 m); 8. Pic de Bassiero $(2903 \mathrm{~m})$.

Fase 3: a) nuevo avance glaciar con la aparición de glaciares cubiertos (con voluminosas morrenas y frentes nítidos) bajo las crestas menos elevadas de los grandes circos y preferentemente en orientación meridional; b) estos glaciares cubiertos presentan longitudes entre 0,8 y $1,7 \mathrm{~km}$ y sitúan sus frentes de a 
2070-2180 m; c) los glaciares bajo las cumbres más elevadas (2750-2900 m) ya no ocupan la totalidad del circo pero mantienen longitudes considerables (2,6-3,0 km); c) el avance también se detecta en pequeños glaciares de circo (longitud de 0,6 - 0,8 km) en orientaciones $\mathrm{N}$ (cumbres a 2500-2600 m) que desarrollan complejos morrénicos (a $2150 \mathrm{~m}$ ) con 2-3 morrenas fronto laterales muy próximas entre sí; d) desaparecen definitivamente los pequeños glaciares en los macizos menos elevados (<2500 m); e) ELA a $2250 \mathrm{~m}$ (diferencia ELA de $615 \mathrm{~m}$ ); f) no se vincula la formación de nuevos glaciares rocosos a esta fase.

Fase 4: a) Nuevo avance glaciar, después del fuerte retroceso posterior a la fase 3, con glaciares en las altas cabeceras de los circos (frentes a $2260 \mathrm{~m}$ ), glaciares cubiertos en orientaciones meridionales con frentes a cotas más elevadas (2300 $2400 \mathrm{~m}$ ) y nuevos glaciares rocosos; b) ELA a 2515 m (diferencia ELA de 355 $\mathrm{m}$ ); d) el grupo 4 de glaciares rocosos es extenso en número de aparatos y en superficie, con frentes a 2120 - 2260 m (DP de 440 m) situándose en los espacios ocupados en la fase anterior por glaciares y glaciares cubiertos; e) el glaciar rocoso más extenso es el de Mines d'Andreuet (Fig. 3 y Tabla 1, punto 18) con $1,2 \mathrm{~km}$ de longitud; f) al final de la Fase 4 se produce un nuevo retroceso glaciar con glaciares blancos derivando en glaciares cubiertos (Clot de Cabanes; Fig. 4 D y Fig. 11), glaciares cubiertos evolucionando hacia formas complejas glaciar cubierto-glaciar rocoso (Pales de Nollerme) y glaciares rocosos con frentes estabilizados y "generaciones" de glaciares rocosos activas, situadas a más altura (polimórficos).

Fase 5: a) Pequeño avance y estabilización glaciar acompañado por la construcción de nuevos glaciares rocosos (grupo 5) que se sitúan ya bajo las paredes de los fondos de circo; b) la ocupación glaciar se limita a los sectores más elevados orientados al $\mathrm{N}$ al pie de los grandes picos (Bassiero, $2903 \mathrm{~m}$; Montsaliente, 2883 m; Agudes, 2707 m) donde en función de las condiciones morfotopográficas (altitud, orientación $\mathrm{N}$, altura de la pared del circo, etc.) se desarrollan pequeños glaciares blancos y glaciares cubiertos (ELA de $2580 \mathrm{~m}$ y diferencia ELA de 290 m); c) el glaciar más representativo de esta fase es el de Cap d'Estany Gerber (Fig. 3, punto 14), que presenta una morrena fronto lateral a $2427 \mathrm{~m}$, una longitud de 1,5 km y 73,7 ha de superficie; d) los frentes de los glaciares rocosos del grupo 5 se sitúan a 2300-2400 m según su orientación (DP de $316 \mathrm{~m}$ ); e) 6 de los 8 nuevos glaciares rocosos se encuentran en el espacio ocupado en la fase anterior por el glaciar del Clot de Cabanes, algunos de ellos son polimórficos y dos claramente de origen glaciogénico (Fig. 3 y Tabla 1, puntos 21 y 22); f) el más extenso es el del Clot de Cabanes (Fig. 3 y Tabla 1, punto 24 y Fig. 12), compuesto por 4 generaciones de glaciares rocosos delimitadas morfológicamente, y una longitud total de $850 \mathrm{~m}$; g) como en la fase anterior, al final de la fase 5 se detecta un retroceso generalizado con algunas estabilizaciones en todos los aparatos, dos pulsaciones en el glaciar cubierto de Puis de Gerber, tres en el glaciar del Cap d'Estany Gerber (Fig. 3, puntos 14 y 15) y las sucesivas generaciones que construyen el glaciar rocoso del Clot de Cabanes (Fig. 12) cada vez a mayor altitud. 


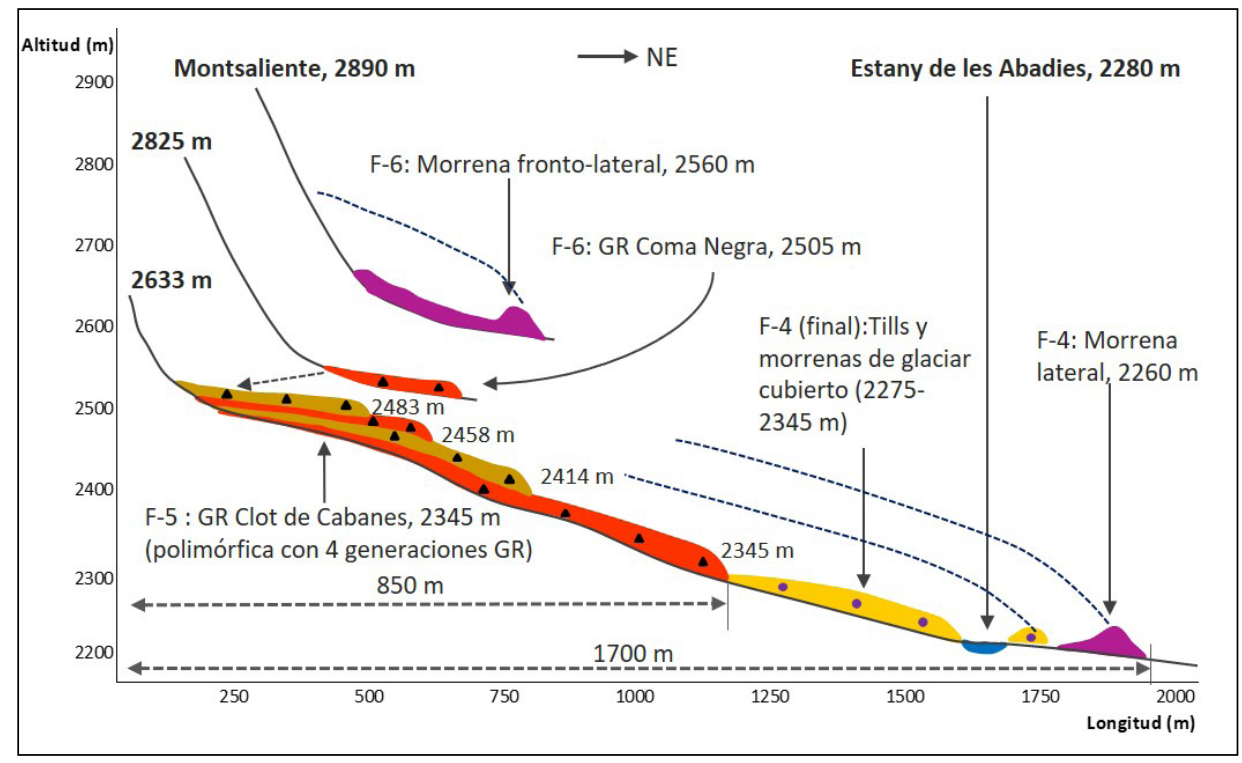

Figura 12. Transecto geomorfológico del Clot de Cabanes. Sucesión de geoformas glaciares y de glaciares rocosos. Se indican las altitudes de los depósitos, la base de los frentes de los glaciares rocosos, las cotas de las crestas y la fase glaciar asociada.

Fase 6: a) Nuevo avance glaciar, con solo dos pequeños glaciares ubicados en las caras $\mathrm{N}$ de los picos más elevados; Cap d'Estany Gerber (Fig. 3, punto 17) y Montsaliente (Fig. 3, punto 16) de 500-600 m de longitud y superficies de 13,2 y 23,9 ha respectivamente. Sus morrenas bien conservadas se sitúan a 2550 $2600 \mathrm{~m}$ (ELA de $2.640 \mathrm{~m}$ y diferencia ELA de $225 \mathrm{~m}$ ); b) a cotas ligeramente inferiores se encuentran cuatro pequeños aparatos, 2 glaciares rocosos y 2 lóbulos protalud (grupo 6; Fig. 3 y Tabla 1, puntos 12,14,15 y 23) con frentes a $2500 \mathrm{~m}$ (DP de $125 \mathrm{~m})$.

Fase 7: a) se trata de una fase "periglaciar" sin glaciares, con funcionalidad actual, representada por un único glaciar rocoso (grupo 7) alojado al pie de la cresta al N del Pic de Bassiero (2903 m), con su frente a $2537 \mathrm{~m}$ (DP de $90 \mathrm{~m}$ ) y una extensión de poco más de 1,5 ha.; b) cercana al glaciar rocoso, una morrena de nevero (a $2640 \mathrm{~m}$ ) y conos de derrubios completan las geoformas periglaciares activas en el circo superior de Cap d'Estany Gerber.

\subsection{Agrupación de glaciares rocosos y fases glaciares}

Después del estudio global de los glaciares rocosos (apartado 5.3) y de la elaboración de una secuencia morfoestratigráfica en la que se relacionan fases glaciares y los grupos de glaciares rocosos vinculados a las mismas, se completa la documentación sobre ellos con una tabla general (Tabla 3) donde se presenta 
una selección de datos básicos ordenados por grupos de glaciares rocosos. Su lectura permite identificar las principales características de estos grupos y las diferencias entre ellos: a) dominio claro de los glaciares rocosos de los grupos 4 y 5 en relación al número de aparatos $(16 ; 55,1 \%)$ y a la superficie acumulada $(112,6$ ha; $60,3 \%)$. Estos grupos además se encuentran en todas las orientaciones y presentan longitudes medias elevadas ( $1^{\mathrm{a}}$ y $3^{\mathrm{a}}$ posición); b) los glaciares rocosos de los grupos más antiguos $(1,2$ y 3 ) situados a cotas más bajas (frentes cercanos a los $2000 \mathrm{~m})$ con 8 aparatos $(27,5 \%)$ ocupan la segunda posición en cuanto a superficie acumulada $(63,6$ ha; $34,0 \%)$; c) los glaciares rocosos pertenecientes a los grupos más recientes $(6$ y 7$)$ son escasos $(5 ; 17,2 \%)$, de reducidas dimensiones y ocupan una superficie aún menor $(10,4$ ha; $5,5 \%)$ y se ubican en las cotas más elevadas; d) en relación a la orientación encontramos en todos los grupos aparatos en todas las orientaciones, dominando como en el conjunto, aquellas más favorables de componente N. Destaca que en los grupos 6 y 7 solo se formaron glaciares rocosos en orientaciones $\mathrm{N}$, siendo aquí la orientación un factor determinante; e) se encuentran glaciares rocosos polimórficos en todos los grupos menos en los más recientes (grupos 6 y 7). En el grupo 4, el $62,5 \%$ de ellos son polimórficos. Ejemplos de glaciares rocosos (monomórficos y polimórficos) en los distintos grupos muestran su desarrollo vertical (altitud de la cumbre, altitud de la raíz, altitud del frente y altitudes de los frentes internos de las sucesivas generaciones que componen los GR polimórficos) (Fig. 13).

Tabla 3. Características de los glaciares rocosos agrupados por fases glaciares (deglaciación). Cronología: OD (Dryas antiguo); YD (Dryas reciente); Hol-t (Holoceno temprano); Hol-m (Holoceno medio); Hol-ta (Holoceno tardío); PEH (Pequeña Edad del Hielo)

\begin{tabular}{|c|c|c|c|c|c|c|c|c|c|c|c|c|}
\hline \multicolumn{7}{|c|}{ VALLE DE LA BONAIGUA: CARACTERISTICAS DE LOS GLACIARES ROCOSOS AGRUPADOS POR FASES GLACIARES } \\
(DEGLACIACION) \\
\hline
\end{tabular}




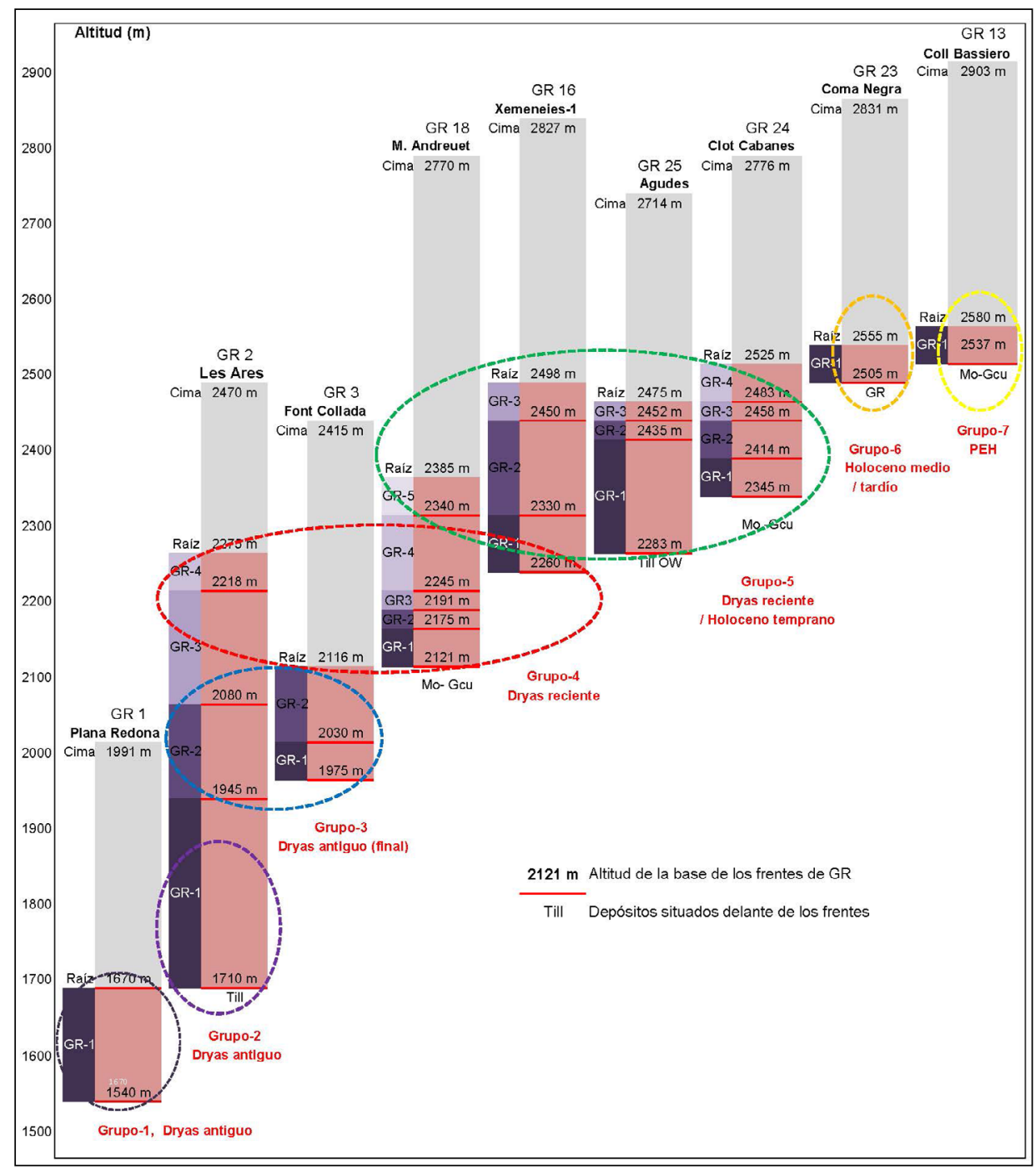

Figura 13. Selección de glaciares rocosos representativos de los grupos GR, identificación de "generaciones" de GR y propuesta cronológica.

\subsection{Las fases glaciares detectadas en relación con el contexto regional pirenaico}

Las primeras fases glaciares (F1, F2 y F3) detectadas en el valle de la Bonaigua las relacionamos, por comparación con otros valles del Pirineo Central, con fases finales del Dryas antiguo (17.5-14.5 ka), correlacionable con la "fase de glaciares de altitud-valle" dentro de la cronología relativa de Bordonau et al. (1992). En el valle de la Bonaigua, durante la primera fase reportada (F1, paleo ELA a $2110 \mathrm{~m})$, los glaciares conservan 
aún la lengua glaciar (paleo glaciar de Cabanes, 5,6 km) pero lejos de las dimensiones alcanzadas en otros valles similares en momentos álgidos del Dryas antiguo. Los glaciares del Pirineo Central en el Dryas antiguo presentan longitudes de 8-15 km en la vertiente $\mathrm{S}$ y hasta $23 \mathrm{~km}$ en la vertiente $\mathrm{N}$, con espesores de 200-300 m (Fernandes et al., 2017; Mahé, 2015; Pallàs et al., 2006; Palacios et al., 2015; Turu et al., 2011). La deglaciación en las vertientes del valle en la F1 facilita la aparición de los primeros glaciares rocosos a cotas bajas (1540 - $1700 \mathrm{~m}$ ), pudiendo situar su génesis entre las fases finales del Dryas antiguo y el inicio de la posterior deglaciación. La formación de glaciares rocosos al final del Dryas antiguo está reportada para el conjunto del Pirineo (Oliva et al., 2019).

Entre la F2 y la F3 se produjo un pronunciado retroceso glaciar al que vinculamos los extensos tills "open work" depositados y el desmembramiento en varios aparatos glaciares de los dos grandes glaciares de circo (Gerber y Cabanes). La progresión y estabilización de los frentes de glaciares cubiertos, a 2070-2180 m, en la entrada de los grandes circos caracteriza a la F3.

Las F4 y F5 son las que se proponen correlacionar con el periodo del Dryas reciente (12.9-11.7 ka) en relación con la "fase de glaciares de altitud-circo" de Bordonau et al. (1992). La paleogeografía de la ocupación glaciar en el valle de la Bonaigua es muy distinta de la de las fases anteriores, con una coexistencia de glaciares de reducidas dimensiones, glaciares cubiertos y glaciares rocosos en el interior de los circos y distribuidos en función de condicionantes morfotopográficos (altitud, orientación y dinámica de las paredes de circo). Estas características en el valle de la Bonaigua son muy similares a las de otras localidades pirenaicas (Oliva et al., 2019), donde en las mejores ubicaciones los glaciares quedan alojados en los circos con la emisión de pequeñas lenguas glaciares (3-3,5 $\mathrm{km}$ de longitud).

La paleo ELA es de 2515 m (F4) y de 2580 m (F5). Los glaciares rocosos de estas dos fases son los más numerosos $(16 ; 55 \%)$ y extensos del conjunto (112,6 ha; 60\%), mayoritariamente "polimórficos" situando sus frentes entre los 2100-2250 m (F4) y 2300 $2400 \mathrm{~m}$ (F5). Las diferencias tanto de la ELA como del LIP en los glaciares rocosos, la distribución espacial de las geoformas y los cambios morfodinámicos detectados en su evolución (transición de glaciar a glaciar cubierto y glaciar rocoso; transición de glaciar cubierto a glaciar rocoso; sucesión de hasta cuatro generaciones de glaciares rocosos) permite individualizar las dos fases y también plantear la posibilidad de que la F5 se extienda temporalmente abarcando al inicio del Holoceno (Holoceno temprano, 11.7-8.0 ka) tal y como indican algunas dataciones de morrenas y glaciares rocosos en macizos similares (Pallàs et al., 2006; Palacios et al., 2017). En los Alpes varios estadios glaciares (Kartell, Kromer) posteriores al Dryas reciente se han reportado como pertenecientes al Holoceno temprano (Ivy-Ochs, 2015; Federici et al., 2016; Hoffman, 2018; Scotti et al., 2017).

La siguiente fase (F6) es de desarrollo espacial muy reducido (pequeños glaciares con morrenas a $2550 \mathrm{~m}$, paleo ELA a $2640 \mathrm{~m}$ y varios glaciares rocosos-lóbulos protalud con frentes a $2500 \mathrm{~m}$ ) pero de gran importancia ya que permite situarla, como hipótesis, en un contexto de "fase Neoglacial" anterior a la PEH. La F6 en el valle de la Bonaigua es correlacionable con la detectada en el macizo Monteixo-Medacorba (Ventura, 2017) 
donde lóbulos protalud activos fosilizan parcialmente a estas morrenas. La F6 es una nueva fase glaciar con glaciares, glacierettes y glaciares rocosos-lóbulos protalud ubicados en los recuencos superiores (orientación $\mathrm{N}$ ), al pie de altas paredes de circo (170-200 m de desnivel), bajo cumbres $(>2800 \mathrm{~m}$ ) en macizos sin glaciares durante la $\mathrm{PEH}$. Las únicas referencias con dataciones numéricas de fases glaciares del Holoceno (en este caso Holoceno medio) en los Pirineos son las morrenas externas del circo de Marboré datadas a 5.1 ka (García Ruiz et al., 2014) y las del circo de Troumouse a 5.1 y 4.6 ka (Gellatly et al., 1992) recogidas en la síntesis de Palacios et al. (2014). En el valle de Arán Fernandes et al. (2017) también sitúan dentro del Holoceno a varios grupos de morrenas localizadas en las zonas más altas de los circos de Saboredo y Bacivèr (entre 2160 y $2590 \mathrm{~m}$ ). Si nuevas dataciones lo corroboran, se trataría de fases glaciares dentro de un Neoglacial en los términos apuntados por Taillefer $(1964,1967)$, de una pequeña fase glacial independiente de las anteriores (a situar entre 5-2.5 ka) después de la completa deglaciación pirenaica en el Optimo Climático Atlántico (7.5-5.5 ka).

En la vertiente meridional de los Alpes y en los Alpes Marítimos, en un contexto geomorfológico similar al pirenaico (altura de cumbres, altura de los frentes morrénicos, paleo ELAs, etc.), se han datado depósitos relacionados con fases holocenas neoglaciares con características similares a las del valle de la Bonaigua: a) Federici et al. (2016) en la cuenca del Gesso identifican morrenas a $2617 \mathrm{~m}$ (de edad Subboreal - Holoceno tardío), situadas por delante de las morrenas de la PEH; b) Finsinger y Ribolini (2001), en el macizo de la Argentera y bajo cumbres de 2800 m, sitúa morrenas (ELA de 2600-2650 m) y glaciares rocosos (frentes a 2500-2550 m) de edad Subboreal; c) Ribolini et al. (2007) describen en el valle de Schiantala morrenas del Subboreal (2.5 ka) remodeladas en glaciares rocosos y afectadas posteriormente por la expansión glaciar de la $\mathrm{PEH}$; d) recientemente, en el macizo de Ecrins-Pelvoux, Le Roy et al. (2017) han realizado dataciones por isótopos cosmogénicos de las últimas morrenas pre-PEH identificando cinco fases o estadios glaciales claramente "neoglaciales" (entre 4.2 ka y $0.9 \mathrm{ka}$ ).

El "intact rock glacier" del Coll de Bassiero (F7) es correlacionable con otros pequeños glaciares rocosos y lóbulos protalud localizados en la misma cuenca de la Noguera Pallaresa, en las caras N de los macizos más altos (>2800 m) de Certascan, Pica d'Estats y Monteixo-Medacorba, con frentes a >2500 m (Ventura, 2016, 2017). En estos macizos durante la PEH se generaron escasos núcleos glaciares (Mont Valier, Pica d'Estats), siendo aquí una fase eminentemente periglaciar. Diversos autores consideran tanto en los Alpes (Cossart et al., 2010) como en los Pirineos (Lampre, 1998) que estos pequeños glaciares rocosos se formaron durante la PEH. Para Serrano y Agudo (2004), Serrano et al. (2011) y Serrano (2014) los actuales glaciares rocosos activos de los Pirineos tienen dos edades, los más pequeños (como el del Coll de Bassiero en el valle de la Bonaigua) estarían originados en la $\mathrm{PEH}$ y los más extensos serian anteriores (dentro del Holoceno medio y tardío, entre 6.0 y $2.5 \mathrm{ka}$ ) reactivándose en el curso de la PEH y permaneciendo aún activos en la actualidad. En relación a la detección y cuantificación de actividad en estas nuevas geoformas inventariadas (Ventura, 2016), recientemente Bataller (2019) detecta movimientos verticales (subsidencia de magnitud decimétrica) en 6 glaciares rocosos al comparar sucesivos modelos digitales de elevación de alta resolución (datos LIDAR) en los macizos de Besiberri-Montardo y Punta Alta- 
Colomèrs, muy cercanos al valle de la Bonaigua. Esta subsidencia puede atribuirse a la degradación del permafrost presente en el interior de los glaciares rocosos. Los primeros resultados (estudio en curso en colaboración con el ICGC) de un análisis interferométrico con imágenes radar (Sentinel-1) muestran también desplazamientos horizontales de 3-5 cm/año en 2 GR de la Punta Harlé (Besiberri-Montardo).

\section{Conclusiones}

La aplicación del método paleogeográfico apoyado en una cartografía geomorfológica detallada ha permitido establecer en el valle de la Bonaigua una secuencia morfoestratigráfica de la deglaciación ordenada en 7 fases (6 glaciares y 1 periglaciar). Los aspectos más destacados del estudio son:

- La caracterización morfológica y sedimentaria de los depósitos ha permitido la diferenciación genética entre tills "open work", morrenas de glaciar cubierto y morrenas de glaciar rocoso, su ocupación espacial y la asignación a fases glaciares distintas.

- En casi todas las fases glaciares dentro de la deglaciación se produce una coexistencia entre glaciares, glaciares cubiertos y glaciares rocosos, en función de la altitud y las características morfotopográficas que dominan cada geoforma (altitud, orientación, dimensión de las paredes de circo, estado de fracturación del roquedo, etc.). Los glaciares rocosos toman protagonismo a medida en que la alta montaña va quedando libre de glaciares. El cambio entre sucesivas fases glaciares también produce en muchas localizaciones la transición o sustitución de un glaciar hacia un glaciar cubierto y de este a glaciares rocoso o directamente la formación de glaciares rocosos bajo las paredes de los circos liberados de hielo glaciar (en concreto entre las fases 3,4 y 5 ).

- Las 7 fases detectadas, por correlación con otras zonas de la cordillera pirenaica (con dataciones absolutas disponibles), se sitúan entre finales del Dryas antiguo (fases 1,2 y 3 ) y la PEH (fase 7). Las fases 4 y 5 se sitúan entre el Dryas reciente y el Holoceno temprano y la fase 6 (bien individualizada) se ubica dentro del Holoceno (Holoceno medio o Holoceno tardío).

- En relación a los glaciares rocosos y lóbulos protalud cabe destacar: 1) la gran amplitud altitudinal (1000 m) y temporal (desde el Dryas antiguo hasta la PEH) que estas geoformas ocupan en el valle de la Bonaigua; 2 ) La localización de glaciares rocosos situados a cotas bajas $(<1600 \mathrm{~m})$ en entornos forestales actualmente; c) la abundante presencia de glaciares rocosos polimórficos atribuibles a su vez fases glaciares distintas, formados por hasta 5 generaciones de grupos de lóbulos en un mismo aparato; d) la clasificación del glaciar rocoso Coll de Bassiero a partir de criterios geomorfológicos de campo como "intact rock glacier".

En el futuro, la continuidad de este estudio deberá dirigirse hacia la elaboración de nuevas secuencias morfoestratigráficas en otras áreas tipo de la cuenca de la Noguera Pallaresa para lograr una secuencia regional (incluyendo la extracción de 
datos paleoclimáticos) y complementarlas mediante dataciones numéricas (martillo de Schmidt) y dataciones absolutas por exposición cosmogénica (DEC) con un especial interés en la datación de probables fases glaciares holocenas.

Nuevos inventarios de glaciares rocosos a nivel de cuenca o la revisión de los existentes son necesarios para disponer de una visión global más exacta, detectar nuevos glaciares rocosos en entornos actualmente forestales e inventariar y monitorizar (por fotogrametría o teledetección radar) aquellos aparatos que presentan aún actividad (presencia de permafrost discontinuo), sobre todo en estos momentos de aceleración del cambio climático.

\section{Referencias}

Baroni, C., Carton, A., Seppi, R. 2004. Distribution and Behaviour of Rock Glaciers in the Adamello-Presanella Massif (Italian Alps). Permafrost and Periglacial Processses 15, 243 259. https://doi.org/10.1002/ppp.497.

Barsch, D. 1996. Rock glaciers. Indicators for the present and former geoecology in high mountain environment. Springer Verlag, Berlin. 331p.

Bataller, F.J. 2019) Pyrenaic rock-glaciers: an airborne and multitemporal lidar monitoring case study in the Besiberri area. Earth ArXiv, https://doi.org/10.31223/osf.io/nxveh.

Benn, D.I., Lehmkuhl, F. 2000. Mass balance and equilibrium-line altitudes of glaciers in highmountain environments. Quaternary International 65-66, 15-29. https://doi.org/10.1016/ S1040-6182(99)00034-8.

Bordonau, J., Serrat, D., Vilaplana, J.M. 1992. Las fases glaciares cuaternarias en los Pirineos. En: A. Cearreta, F.M. Ugarte (Eds.), The Late Quaternary in the Western Pyrenean Region. Servicio Editorial Universidad del País Vasco, Bilbao, pp. 303-312.

Cossart, E., Fort, M., Bourles, D., Carcaillet, J., Perrier, R., Siame, L., Braucher, R. 2010. Climatic significance of glacier retreat and rockglaciers re-assessed in the light of cosmogenic and weathering rind thickness in Clarée valley (Briançonnais, French Alps). Catena 80, 204-219. https://doi.org/10.1016/j.catena.2009.11.007.

De Verneuil, De Keyserling 1861. Coupes du versant meridional des Pyrénées. Bulletin de la Societé Géologique de France 18, 341 pp.

Durocher, J. 1841. Sur les traces de phénoménes diluviens qui s'observent dans les Pyrénées". Comptes Rendues de l'Académie des Sciences, 13, 902-903.

Federici, P., Ribolini, A., Spagnolo, M. 2016. Glacial history of the Maritime Alps from the Last Glacial Maximum to the Little Ice Age. En: P.D. Hughes, J.C. Woodward (Eds.), Quaternary Glaciation in the Mediterranean Mountains. Geological Society, London, Special Publications, 433. http://doi.org/10.1144/SP433.9.

Fernandes, M., Oliva, M., Palma, P., Ruíz-Fernandez, J., Lopes, L. 2017. Glacial stages and post-glacial environmental evolution in the Upper Garonne valley, Central Pyrenees. Science of Total Environment 584-585 (2017), 1282-1299. https://doi.org/10.1016/j. scitotenv.2017.01.209.

Fernandes, M., Palma, P., Lopes, L., Ruiz-Fernández, J., Pereira, P., Oliva, M. 2017. Spatial distribution and morphometry of permafrost-related landforms in the Central Pyrenees and associated paleoclimatic implications. Quaternary International. https://doi.org/10.1016/j. quaint.2017.08.071.

Finsinger, W., Ribolini, A. 2001. Late glacial to Holocene deglaciation of the Colle del Vei del Bouc-Colle del Sabbione Area (Argentera Massif, Maritime Alps, Italy-France). Geografia 
Fisica e Dinamica Quaternaria 24, 141-156. http://www.glaciologia.it/wp-content/uploads/ Abstracts/Abstract_24_2/02-Finsinger_141-156.pdf.

Fontboté, J.M., Solé Sabarís, L., Alimen, H. 1957. Livret guide de l'excursion N1, Pyrénées. VIème-VIIème journées. V Congreso Internacional INQUA. Madrid-Barcelona, pp. 65-74

Fernández-Fernández, J.M., Palacios, D., García-Ruiz, J.M., Andrés, N., Schimmelpfennig, I., Gómez-Villar, A., Santos-González, J., Álvarez-Martínez, J., Arnáez, J., Úbeda, J., Léanni, L., ASTER Team 2017. Chronological and geomorphological investigation of fòssil debris-covered glaciers in relation to deglaciation processes: A case study in the Sierra de la Demanda, northern Spain. Quaternary Science Reviews 170, 232-249. https://doi. org/10.1016/j.quascirev.2017.06.034.

Frauenfelder, R., Kääb, A. 2000. Towards a paleoclimatic model of rock-glacier formation in the Swiss Alps.Annals of Glaciology31,281-286.https://doi.org/10.3189/172756400781820264.

García Ruíz, J.M., Palacios, D., de Andrés, N., Valero-Garcés, B., López-Moreno, J.I., Sanjuán, Y., 2014. Holocene and "Little Ice Age" glacial activity in the Marboré Cirque, Monte Perdido Massif, Central Spanish Pyrenees. The Holocene 24 (2), 1439-1452. https://doi. org/10.1177/0959683614544053.

García Ruíz, J.M., Palacios, D., González-Sampériz,P., de Andrés, N., Moreno, A., Valero-Garcés, B., Gómez-Villar, A. 2016. Mountain glacier evolution in the Iberian Peninsula during the Younger Dryas. Quaternary Science Reviews 138, 16-30. https://doi.org/10.1016/j. quascirev.2016.02.022.

García Sainz, L. 1935. Morfología glaciar y preglaciar de la región de la Noguera. Boletín de la Sociedad Geográfica Nacional 75, 64-130.

Gellatly, A.F., Grove J.M., Switsur, V.R. 1992. Mid-Holocene glacial activity in the Pyrenees. The Holocene 2 (3), 266-270. https://doi.org/10.1177/095968369200200309.

Gómez-Villar, A., González-Gutiérrez, R.B., Redondo-Vega, J.M., Santos-González, J. 2011. Distribución de glaciares rocosos relictos en la Cordillera Cantábrica. Cuadernos de Investigación Geográfica 37 (2), 49-80. https://doi.org/10.18172/cig.1256.

González Trueba, J.J. 2007. Geomorfología del Macizo Central de los Picos de Europa (contiene mapa geomorfológico 1:25.000). Madrid, Organismo Autónomo Parques Nacionales. Ministerio de Medio Ambiente. 230 pp.

Hamelin, L.E. 1958. Materiaux de geomorphologie periglaciaire dans l'Espagne du Nord. Revue Géographique des Pyrénées et du Sud-Ouest 29, 241-256. https://doi.org/10.3406/ rgpso.1958.4695.

Haeberli, W. 1985. Creep of mountain permafrost: internal structureand flow of alpine rock glaciers. Mitteilungen der Versuchsanstalt für Wasserbau, Hydrologie und Glaziologie, ETHZürich, 1-119.

Hofmann, F.M. 2018. Glacial history of the upper Drac Blanc catchment (Écrins massif, French Alps). E\&G Quaternary Science Journal 67, 37-40. https://doi.org/10.5194/ egqsj-67-37-2018.

Institut Cartogràfic i Geològic de Catalunya, ICGC (2007). Mapa geològic comarcal de Catalunya 1:50.000: Pallars Sobirà. Barcelona.

Institut Cartogràfic i Geològic de Catalunya, ICGC (2015). Mapa de les unitats estructurals majors de Catalunya 1:1.000.000. Barcelona.

Institut Cartogràfic i Geològic de Catalunya, ICGC (2016). Model d'Elevacions del Terreny de Catalunya 2x2 metres (MET-2) v1 .0. Especificacions tècniques. Barcelona.

Ivy-Ochs, S. 2015. Glacier variations in the european Alps at the end of the Last Glaciation. Cuadernos de Investigación Geográfica 41 (2), 295-315. https://doi.org/10.18172/cig.2750.

Kellerer-Pirklbauer, A., Lieb, G.K., Kleinferchner, H. 2012. A new rock glacier inventory of the Eastern european Alps. Austrian Journal of Earth Sciences 105 (2), 78-93. 
Lambiel, C., Reynard, E. 2003. Cartographie de la distribution du pergélisol et datation des glaciers rocheux dans la région du Mont Gelé (Valais). Physische Geographie 41, 91-103.

Lampre, F. 1994. La línea de equilibrio glacial y los suelos helados en el macizo de la Maladeta (Pirineo Aragonés): Evolución desde la Pequeña Edad del Hielo y situación actual. En: C. Martí Bono, J.M. García Ruíz (Eds.), El Glaciarismo surpirenaico: Nuevas aportaciones. Ed. Geoforma, Logroño, pp. 125-142.

Lampre, F. 1998. Estudio geomorfológico de Ballibierna (Macizo de la Maladeta-Pirineo Aragonés). Publicaciones del Consejo de Protección de la Naturaleza de Aragón. Zaragoza, $200 \mathrm{pp}$.

Le Roy, M., Deline, P., Carcaillet, J., Schimmelpfennig, I., Ermini, M., ASTER Team 2017. 10Be exposure dating of the timing of Neoglacial glacier advances in the Ecrins-Pelvoux massif, southern French Alps. Quaternary Science Reviews 178, 118-138. https://doi.org/10.1016/j. quascirev.2017.10.010.

Lilleoren, K., Etzelmüller, B. 2011. A regional inventory of rock glaciers and ice-cored moraines in Norway. Geografiska Annaler, Serie A, Physical Geography 93, 175-191. https://doi. org/10.1111/j.1468-0459.2011.00430.x.

Mahé, C. 2015. Paléogéographie et chronologie des paléoenglacements tardiglaciaires pyrénéens (Mémoire de Master). Université Paris IV Sorbonne. 149 pp.

Martí, M., Serrat, D. 1990. Los glaciares rocosos del Pirineo catalán: primeros resultados. Actas I Reunión Nacional de Geomorfología, S.E.G., 1, Teruel, pp. 191-201.

Martí, M., Serrat, D. 1992. Les glaceres rocalloses al Parc Nacional d'Aigüestortes i Estany de Sant Maurici i la seva área d'influència. En: La investigació al Parc Nacional d'Aigüestortes $i$ Estany de Sant Maurici. Segones jornades sobre recerca (ponències). Espot, 22 al 25 de octubre de 1991, pp. 43-51. Direcció General del Medi Natural. Departament d'Agricultura, Ramaderia i Pesca, Generalitat de Catalunya

Martí, M. 1994. L'ocupació de l'espai per les glaceres rocalloses. L'exemple de Cometes de Moró. En: La investigació al Parc Nacional d'Aigüestortes i Estany de Sant Maurici. Terceres jornades sobre recerca (ponències). Boí (1993), 19 pp. Direcció General del Medi Natural. Departament d'Agricultura, Ramaderia i Pesca, Generalitat de Catalunya.

Martín-Parra, L.M., Bellido, F., Rodríguez Fernández, L.R., Suárez Rodríguez, A., y Zamora, P. (2016). Mapa Geológico, del Mapa Geológico de España a E. 1:50.000, № 181 (Esterri D’Aneu). Segunda Serie MAGNA. IGME. Madrid.

Martín Vide, J., Brunet, M., Prohom, M., Rius, A. 2011. Els climes de Catalunya. Present i tendències recents. En: El canvi climàtic a Catalunya, 43-72.

Masachs, V., Montoriol, J. 1961. Las formes periglaciares del Port de la Bonaigua y del circo de Els Erculls (Pirineo de Lérida). Speleon 12 (3-4), 23-38.

Matthews, J.A., Wilson, P., Mourne, R.W. 2017. Landforms transition from pronival ramparts to moraines and rock glaciers: a case study from the Smorbotn cirque, Romsdalsalpane, Southern Norway. Geografiska Annaler: Series A, Physical Geography 99:1,15-37. https:// doi.org/10.1080/04353676.2016.1256582.

Monnier, S. 2004. Identification, caractérisation et distribution spatiale des glaciers-rocheux dans la haute vallée de l'Arc (Alpes françaises du Nord). Géomorphologie: Relief, Processus, Environnement 10 (2), 139-155. https://doi.org/10.3406/morfo.2004.1210

Nussbaum, F. 1934. Die See der Pyrenäen. Mitt. der Natur. Gesensch, 184 pp. Berna.

Nussbaum, F. 1956. Observations morphologiques dans la région de la Noguera Pallaresa. Pirineos (39-42), 57-97.

Oliva, M., Serrano, E., Gómez-Ortiz, A., González-Amuchástegui, M.J., Nieuwendam, A., Palacios, D., Pérez-Alberti, A., Pellitero-Ondicol, R., Ruiz-Fernández, J., Valcárcel, M., Vieira, G., Antoniades, D. 2016. Spatial and temporal variability of periglaciation of the 
Iberian Peninsula. Quaternary Science Reviews 137, 176-199. https://doi.org/10.1016/j. quascirev.2016.02.017.

Oliva, M., Palacios, D., Fernández-Fernández, J.M., Rodríguez-Rodríguez, L., García-Ruíz, J.M., Andrés, N., Carrasco, R.M., Pedraza, J., Pérez-Alberti, A., Valcárcel, M., Hughes, P.D. 2019. Late Quaternary glacial phases in the Iberian Peninsula. Earth-Science Reviews 192, 564600. https://doi.org/10.1016/j.earscirev.2019.03.015.

Ortuño, M. 2008. Deformación activa en el Pirineo Central: la falla Norte de la Maladeta y otras fallas activas. Tesis doctoral, 346 pp. Risknat, Departament de Geodinàmica i Geofísica, Facultat de Geologia, Universitat de Barcelona.

Palacios, D., de Andrés, N., López-Moreno, J.I., García-Ruiz, J.M. 2014. The deglaciation of the Pyrenees: from the Oldest Dryas to the Litlle Ice Age. Geophysical Research Abstracts, 16, EGU2014-11066. EGU General Assembly, Viena.

Palacios, D., de Andrés, N., López-Moreno, J.I., García-Ruiz, J.M. 2015. Late Pleistocene deglaciation in the upper Gállego Valley, central Pyrenees. Quaternary Research 83 (3), 397-414. http://dx.doi.org/10.1016/j.yqres.2015.01.010.

Palacios, D., Andrés, N., Gómez-Ortiz, A., García-Ruiz, J.M. 2016. Evidence of glacial activity during the Oldest Dryas in the mountains of Spain. En: P.D. Hughes, J.C. Woodward (Eds.), Quaternary Glaciation in the Mediterranean Mountains. Geological Society, London, Special Publications 433, http://doi.org/10.1144/SP433.10.

Palacios, D., García-Ruiz, J.M. de Andrés, N., Schimmelpfennig, I., Campos, N., Léanni, L., ASTER Team 2017. Deglaciation in the central Pyrenees during the Pleistocene-Holocene transition: Timing and geomorphological significance. Quaternary Science Reviews 162, 111-127. https://doi.org/10.1016/j.quascirev.2017.03.007.

Pellitero, R. 2014. Geomorphology and geomorphological landscapes of Fuentes Carrionas. Journal of Maps 10 (2), 313-323, https://doi.org/10.1080/17445647.2013.867822.

Pallàs, R., Rodés, A., Braucher, R., Carcaillet, J., Ortuño, M., Bordonau, J., Bourlès, D., Vilaplana, J.M., Masana, E., Santanach, P. 2006. Late Pleistocene and Holocene glaciation in the Pyrenees:a critical review and new evidence from $10 \mathrm{Be}$ exposure ages, southcentral Pyrenees. Quaternary Science Reviews 25, 2937-2963. https://doi.org/10.1016/j. quascirev.2006.04.004.

Porter, S.C. 1975. Equilibrium-line altitudes of late Quaternary glaciers in the Southern Alps, New Zealand. Quaternary Research 5, 27-47. https://doi.org/10.1016/0033-5894(75)90047-2 .

Ribolini, A., Chelli, A., Guglielmin, M., Pappalardo, M. 2007. Relationships between glacier and rock glacier in the Maritime Alps, Schiantala Valley, Italy. Quaternary Research, 68, 353363. https://doi.org/10.1016/j.yqres.2007.08.004.

Scapozza, C. 2015. Evolution des glaciers et du pergélisol depuis le dernier maximum glaciaire dans la región du Mont Gelé-Mont Fort (Alpes Valaisannes, Suisse): chronologie, modalités de la dernière déglaciation et datations des âges d'exposition a l'aide du Marteau de Schmidt. Quaternaire 26 (2), 141-173. https://doi.org/10.4000/ quaternaire.7250.

Scapozza, C., Mari, S. 2010. Catasto, caratteristiche e dinàmica dei rock glaciars delle Alpi Ticinesi. Bolletino de la Società Ticinese di Scienze Naturali 98, 15-29. http://repository. supsi.ch/2152/1/Scapozza-Mari_Boll.-STSN2010.pdf.

Schoeneich, P. 1993. Comparaison des systèmes de légendes français, allemande et Suisse principes de la légende IGUL. En: Cartographie geomorphologique - Cartographie des risques. Institut de Géographie de Lausanne, Série Travaux et recherches 9, 15-24.

Scotti, R., Brandinoni, F., Crosta, G.B., Cola, G. 2017. Time constraints for post-LGM landscape response to deglaciation in Val Viola, Central Italian Alps. Quaternary Science Reviews 177, 10-33. https://doi.org/10.1016/j.quascirev.2017.10.011. 
Serrano, E., Agudo, C., González Trueba, J.J. 2002. La deglaciación de la alta montaña. Morfología, evolución y fases morfogenéticas glaciares en el macizo de Posets (Pirineo Aragonés). Cuaternario y Geomorfología 16 (1-4), 111-126. http://tierra.rediris.es/ CuaternarioyGeomorfologia/images/vol16/Cuaternario16(1-4)_11.pdf.

Serrano, E., Agudo, C. 2004. Glaciares rocosos y deglaciación en la alta montaña de los Pirineos aragoneses (España). Boletín de la Real Sociedad Española de Historia Natural (Sec. Geol.) 99 (1-4), 159-172.

Serrano, E., González Trueba, J.J. 2004. El método AAR para la determinación de paleo-ELAs: Análisis metodológico y aplicación en el macizo de Valdecebollas (Cordillera Cantábrica). Cuadernos de Investigación Geográfica 30, 7-34. https://doi.org/10.18172/cig.1133.

Serrano, E., Morales, C., González Trueba, J.J., Martin, R. 2009. Cartografía del permafrost de montaña en los Pirineos españoles. Finisterra 87, 45-54. https://doi.org/10.18055/Finis1376.

Serrano, E., González Trueba, J.J., San José, J.J. 2011. Dinámica, evolución y estructura de los glaciares rocosos de los Pirineos. Cuadernos de Investigación Geográfica 37, 145-170. https://doi.org/10.18172/cig.1260.

Serrano, E. 2014. Glaciares rocosos. Controversias y certidumbres. En: A. Gómez Ortiz, F. Salvador Franch, M. Oliva Franganillo, M. Salvà Catarineu (Eds.). Avances, métodos y técnicas en el estudio del periglaciarismo. Publicacions i Edicions de la Universitat de Barcelona. Barcelona, pp. 115-134.

Serrat, D. 1979. Rock glacier morainic deposits in the Eastern Pyrenees. En: Ch. Schlüchter (Ed.), Moraines and varves, Ed. A.A. Balkema, Rotterdam, pp. 93-100.

Serrat, D., Bordonau, J., Bru, J., Furdada, G., Gómez Ortiz, A., Martí, J., Salvador, F., Ventura, J., Vilaplana, J.M. 1994. Síntesis cartográfica del glaciarismo surpirenaico oriental. En: C. Martí Bono, J.M. García Ruíz (Eds.), El Glaciarismo surpirenaico: Nuevas aportaciones. Geoforma Ediciones, Logroño, pp. 9-15.

Solé Sabarís, L. 1936. Els llacs dels Pirineus segons Nussbaum. Barcelona, Butlletí de la Institució Catalana d'Història Natural 36, 107-115.

Suárez Rodríguez, A., Alonso, V. (2005). Aigüestortes and lake Sant Maurici National Park Geomorphological Map (Pyrenees, NE Spain), E:1:25.000. VI ${ }^{\circ}$ International Conference on Geomorphology. Vol. Abstracts, p.404. Zaragoza.

Suárez Rodríguez, A. (2016). Mapa Geomorfológico del Mapa Geológico de España a E. 1:50.000, $\mathrm{N}^{\mathrm{o}} 181$ (Esterri D’Aneu). Segunda Serie MAGNA. IGME Madrid.

Taillefer, F. 1964. Le modelé post-wurmien des hautes montagnes françaises. Revue Géographique des Pyrénées et du Sud-Ouest 35 (2), 129-138. https://doi.org/10.3406/rgpso.1964.2138.

Taillefer, F. 1967. Le neoglaciaire pyrenéen. Melanges O. Tulippe. Ed. J. Ducalot. Gemblox, 134148.

Turu, V., Vidal-Romaní, J.R., Fernández-Mosquera, D. 2011. Dataciones con isótopos cosmogénicos (10Be): El "LGM" Last Glacial Maximum y "the Last Termination" en los valles del Gran Valira y la Valira del Nord (Principado de Andorra, Pirineos Orientales). En: V. Turu, A. Constante (Eds.), El Cuaternario en España y áreas afines, Avances en 2011. Resúmenes XIII Reunión Nacional de Cuaternario, Andorra la Vella, pp. 49-50.

Ventura, J. 1983. Geomorfologia glaciar de la Vall de Espot, Pallars Sobirà - Pirineu Central (Tesis de Licenciatura). Universitat de Barcelona. Dept. de Geografía, Barcelona, 231 pp.

Ventura, J. 1992. Geomorfologia glaciar de les valls de la Bonaigua, Son i els sectors occidentals de la cubeta d'Esterri d'Àneu. En: La investigació al Parc Nacional d'Aigüestortes i Estany de Sant Maurici. Segones jornades sobre recerca (ponències). Espot, 22 al 25 de octubre de 1991, pp. 23-41. Direcció General del Medi Natural. Departament d'Agricultura, Ramaderia i Pesca, Generalitat de Catalunya. 
Ventura, J. 2010. Geomorfologia de Les Planes de Son i La Mata de València. L' influencia del modelat glacial i periglacial. En: Els sistemes naturals de Les Planes de Son i la Mata de València. Treballs de la Institució Catalana d' Història Natural 16, 77-125.

Ventura, J. 2016. Identificación e inventario de potenciales glaciares rocosos activos en los Pirineos mediante fotointerpretación en visores cartográficos 2D y 3D: Primeros resultados. Polígonos 28, 95-121. https://doi.org/10.18002/pol.v0i28.4289.

Ventura, J. 2017. Identificación de fases glaciares durante la deglaciación en el Macizo de MonteixoMedacorba (Pirineo Central, Lleida). En: J. Ruíz-Fernández, C. García-Hernández, M. Oliva, C. Rodríguez-Pérez, D. Gallinar (Eds.), Ambientes periglaciares: Avances en su estudio, valoración patrimonial y riesgos asociados. Servicio de Publicaciones de la Universidad de Oviedo, pp. 137-146.

Verneuil, E.P., von Keyserling, A. 1861. Coupes du versant meridional des Pyrénées. Bulletin de la Société Géologique de France, 18, p. 341

Whalley, W.B. 2009. On the interpretation of discrete debris accumulations associated with glaciers with special reference to the British Isles. En: J. Knight, S. Harrison (Eds.): Periglacial and Paraglacial Processes and Environments. The Geological Society, London, Special Publications, 320, 85-102. https://doi.org/10.1144/SP320.7.

Whalley, W.B., Azizi, F. 2003. Rock glaciers and protalus landforms: analogous forms and ice sources on Earth and Mars. Journal of Geophysical Research Planets 108 (E4) 8032. https:// doi.org/10.1029/2002JE001864.

Wrethman, A.C. 2018. Reconstructing the glacial history of the upper Glen Dee. Dundee. University of Dundee, $80 \mathrm{pp}$.

Zandvliet, J. 1960. The Geology of the Upper Salat and Pallaresa Valleys, Central Pyrenees France/Spain. Leidse Geologische Mededelingen 25, 1-127. 